\title{
La libertà di stampa nella raccolta di informazioni, l'accesso all'informazione e la protezione dei whistleblower in base all'art. 10 CEDU e degli standard del Consiglio d'Europa
}

Dirk Voorhoof, Ghent University (Belgio)

\section{INTRODUZIONE ${ }^{1}$}

Circa 33 anni fa, la Dichiarazione sulla libertà di espressione e di informazione del Consiglio d'Europa ha evidenziato un forte attaccamento ai principi della libertà di espressione e di informazione "quali elemento fondante di una società democratica e plurale". Tale Dichiarazione, adottata il 29 aprile 1982 (Dichiarazione del 1982) ha posto l'accento, soprattutto in riferimento all'art. 10 della Convenzione europea dei diritti dell'uomo (CEDU), sulla:

Protezione del diritto di chiunque, a prescindere dalle frontiere nazionali, di esprimersi, di ricercare e ottenere informazioni e idee, indipendentemente dalla loro fonte, così come di divulgarle secondo le condizioni previste dall'art. 10 della Convenzione europea dei diritti dell'uomo.

La Dichiarazione del 1982 ha inoltre sottolineato che, nel campo dell'informazione e dei mass media, uno degli obiettivi consiste nel:

Perseguimento di una politica sull'informazione libera, incluso l'accesso all'informazione, al fine di potenziare la comprensione di questioni politiche, sociali, economiche e culturali e la capacità di discuterne liberamente.

1 Il presente capitolo si basa sulla ricerca e alcune precedenti bozze di paper e pubblicazioni, in particolare: Voorhoof D. (2015a); Voorhoof D. (2014a); e Voorhoof D. e Humblet P. (2013). Un riepilogo, comprendente anche una banca dati relativa alla casistica principale della CEDU sulla libertà d'espressione, sui media e sul giornalismo è disponibile in versione $e$-book, pubblicato dall' Osservatorio europeo dell'audiovisivo (Strasburgo: Iris): http://www.obs.coe.int/documents/205595/2667238/ IRIS+Themes+III+(nal+9+December+2013).pdf/2e748bd5-7108-4ea7-baa6-59332f 885418. Vedi anche Voorhoof D. (2015b). 
Un anno prima, il 25 novembre 1981, il Comitato dei Ministri del Consiglio d'Europa avevo adottato una raccomandazione (Raccomandazione del 1981 ) in cui si raccomandava agli Stati membri in maniera più esplicita, ma senza riferimento all'art. 10 della CEDU, di riconoscere nelle proprie giurisdizioni il diritto per chiunque di ottenere, su richiesta, informazioni detenute dalle pubbliche autorità, da altri organi legislativi e autorità giudiziarie. $^{2}$

Nonostante la Raccomandazione del 1981 e la Dichiarazione del 1982 si riferissero al diritto "di cercare informazioni", al diritto "di accedere alle informazioni" e al diritto "di avere accesso agli atti pubblici", va osservato che il testo dell'art. 10 della CEDU stesso non fa riferimento, nel garantire il diritto alla libertà di espressione, a un tale diritto. ${ }^{3}$ Ciononostante, il testo della Dichiarazione del 1982 e altri documenti pubblicati dal Consiglio d'Europa spiegano l'importanza del diritto di cercare informazioni e del diritto di accesso agli atti pubblici e la necessità di includerli o inserirli nel diritto alla libertà di espressione. È stato evidenziato e ripetuto che la trasparenza è essenziale in una società democratica e che un ampio accesso alle informazioni su materie di interesse generale consente al pubblico di avere un'adeguata visione sullo stato della società in cui esso vive e di formarsi un'opinione critica sulla stessa. ${ }^{4}$ Fin dal 1979, la Corte europea dei diritti dell'uomo (la Corte) ha riconosciuto, ripetuto ed evidenziato nella propria giurisprudenza "il diritto del pubblico di essere adeguatamente informato" su questioni di interesse per la società. ${ }^{5}$

Nella propria Raccomandazione del 21 febbraio 2002, il Comitato dei Ministri del Consiglio d'Europa ha compiuto un ulteriore passo in avanti. Non ha solamente confermato il principio secondo il quale gli Stati membri dovrebbero garantire il diritto di tutti di accedere, su richiesta, ai documenti pubblici detenuti dalle pubbliche autorità, ma ha stabilito che l'ac-

2 Comitato dei Ministri del Consiglio d'Europa (1981).

3 Vedi invece l'art. 19 del Patto delle Nazioni Unite sui diritti civili e politici che richiede agli Stati di garantire il diritto alla libertà di espressione, il quale comprende il diritto di ricercare, ricevere e divulgare informazioni e idee indipendentemente dai confini. Il diritto di ricercare e ricevere informazioni e idee include "il diritto di accesso alle informazoni in possesso di enti pubblici”: Comitato per i diritti umani, Commento generale n. $34 \mathrm{CCPR} / \mathrm{C} / \mathrm{GC} / 34$, sulla libertà di opinione e di espressione (Articolo 19 PIDCP), 12 settembre 2011.

4 Darbishire H. (2015). Vedi anche Hins W. e Voorhoof D. (2007).

5 Sunday Times c. Regno Unito (n. 1) e più recentemente Morice c. Francia, \$\$ 150-153 e Erla Hlynsdttir c. Islanda (n. 3), $\$ 62$, in cui la Corte ribadì: "Non solo la stampa ha il dovere di divulgare informazioni e idee" relative alle questioni di interesse pubblico, "ma anche il pubblico ha il diritto di riceverle". 
cesso alle informazioni debba altresì includere un comportamento proattivo da parte delle pubbliche autorità per rendere più facilmente accessibili le informazioni di pubblico interesse. Infatti, ha anche statuito che gli Stati membri dovrebbero considerarlo un dovere a cui deve ottemperare una pubblica autorità

su propria iniziativa e qualora sia appropriato adottare le misure necessarie per rendere pubbliche le informazioni da essa detenute quando la diffusione di tali informazioni corrisponde all'interesse di promuovere la trasparenza della pubblica amministrazione e l'efficienza all'interno delle amministrazioni o quando essa esorterà ad una partecipazione informata da parte del pubblico in questioni di pubblico interesse. ${ }^{6}$

La Corte si è, tuttavia, dimostrata molto riluttante nel riconoscere il diritto di accesso alle informazioni, specialmente il diritto di accesso ai documenti detenuti dalle autorità, così come nel rendere tale diritto coercibile ai sensi dell'art. 10 della CEDU. Solamente qualche anno fa la Corte ha cominciato, non senza esitazioni, a mutare il proprio approccio e a includere, fino a una certa misura, un diritto di accesso ai documenti pubblici in relazione con il diritto di esprimere e ottenere informazioni e idee. Specialmente a partire dalle sentenze Táraság a Szabadságjogokért (TASZ) c. Ungheria e Kenedi c. Ungheria nel 2009, la giurisprudenza della Corte ha cominciato a riconoscere e a sviluppare un diritto di accesso ai pubblici documenti ricondotto all'ambito dell'art. 10 della CEDU. ${ }^{7}$ Contemporaneamente, venne promulgata, nel 2009, la Convenzione europea sull'accesso ai documenti ufficiali, la quale faceva, a sua volta, riferimento alle Raccomandazioni del Consiglio d'Europa del 1981 e del 2002 e all'art. 10 della CEDU. La Convenzione Europea sull'accesso ai pubblici documenti del 2009 stabilisce che:

Qualsiasi controparte dovrebbe garantire il diritto di tutti, senza discriminazioni di alcun tipo, di accedere, su richiesta, ai documenti ufficiali detenuti dalle pubbliche autorità. Qualsiasi controparte dovrebbe adottare le misure necessarie all'interno del proprio ordinamento per

6 Comitato dei Ministri del Consiglio d'Europa (1981) and Comitato dei ministri del Consiglio d'Europa (2002).

7 Társaság a Szabadságjogokért c. Ungheria and Kenedi c. Ungheria. 
dare efficacia alle previsioni per l'accesso ai documenti ufficiali enunciate nella Convenzione. ${ }^{8}$

Il riconoscimento, da parte della Corte, sulla base dell'art. 10 CEDU, di un diritto di accesso ai documenti detenuti dalle pubbliche autorità implica che gli Stati membri, i loro organi amministrativi e le autorità giudiziarie non possano più determinare pienamente l'ambito e l'applicazione pratica delle loro normative nazionali che garantiscono il diritto di accesso ai pubblici documenti, in quanto la garanzia pratica ed effettiva di tale diritto è ora al vaglio della Corte. Anche senza che la Convenzione sull'accesso ai documenti ufficiali del 2009 entrasse in vigore, l'art. 10 della CEDU e la giurisprudenza della Corte formano una cornice giuridicamente vincolante per l'attuazione del diritto di accesso ai pubblici documenti negli Stati membri del Consiglio d'Europa. Qualsiasi interferenza con il diritto di accesso ai pubblici documenti deve essere giustificata in quanto necessaria in una società democratica all'interno dell'ambito di applicazione dell'art. 10(2) della CEDU, eventualmente in combinazione con l'art. 6 della CEDU (diritto a un equo processo) e all'art. 13 della CEDU (diritto a un ricorso effettivo). Nella propria casistica recente, la Corte ha evidenziato che:

nei casi in cui il ricorrente era un singolo giornalista ed un difensore dei diritti umani, la Corte ha ritenuto che la ricerca di informazioni rappresenta una fase preparatoria essenziale del giornalismo e un elemento intrinseco e protetto della libertà di stampa.

La Corte ribadisce poi che:

gli ostacoli creati con lo scopo di impedire l'accesso a informazioni di pubblico interesse potrebbero scoraggiare gli operatori del settore mediatico o di settori ad esso collegati dal perseguire tali obiettivi. Di conseguenza, essi potrebbero non essere più in grado di svolgere il loro

8 Convenzione del Consiglio d'Europa sull'accesso a documenti ufficiali (CETS n. 205), art. 2. Accessibile al link: www.conventions.coe.int/Treaty/Commun/QueVo ulezVous.asp? $\mathrm{NT}=205 \& \mathrm{CM}=8 \& \mathrm{DF}=24 / 09 / 2012 \& \mathrm{CL}=\mathrm{ENG}$.

Tuttavia, questa convenzione non è ancora entrata in vigore: solamente sei stati membri lo hanno ratificato (Bosnia e Erzegovina, Ungheria, Lituania, Montenegro, Norvegia and Svezia), e le ratifiche necessarie affinchè vi sia l'entrata in vigore del patto sono 10. Per un'analisi degli sviluppi del diritto di informazione a livello nazionale, vedi Coliver S. (2015). 
ruolo vitale di pubblici guardiani e verrebbe lesa la loro abilità di procurare informazioni accurate ed affidabili. ${ }^{9}$

Tale ampliamento del campo di applicazione del diritto alla libertà di espressione e di informazione va di pari passo con un altro sviluppo nella casistica della Corte che ha contribuito a garantire gradualmente più trasparenza nella società su materie di pubblico interesse proteggendo, di nuovo ex art. 10 CEDU, i diritti dei c.d. "whistleblower", i quali diffondono o "svelano" informazioni ai media. Il diritto alla libertà di espressione dei whistleblower è stato riconosciuto ed efficacemente garantito da parte della Corte all'interno della propria casistica degli ultimi anni, in particolare in seguito alla sentenza della Grande Camera della Corte nel caso Guja c. Moldavia del 2008. ${ }^{10}$ Un tale mutamento verso la previsione di garanzie adeguate per la protezione dei whistleblower si nota anche nella Raccomandazione del Consiglio d'Europa $\operatorname{Rec}(2014) 7$ sulla protezione dei whistleblower (Raccomandazione del 2014), la quale stabilisce che:

gli individui che riportano o svelano informazioni su minacce o danni per il pubblico interesse ("whistleblowers") possono contribuire a rafforzare la trasparenza e la responsabilità democratica. ${ }^{11}$

Di conseguenza, i whistleblower possono invocare il loro diritto alla libertà di espressione quando rivelano informazioni ai media. L'Assemblea parlamentare del Consiglio d'Europa ha ribadito, in una Risoluzione del 23 giugno 2015, l'importanza della casistica della Corte nel sostenere la libertà di parola e la protezione dei whistleblower. Ha fatto appello alla creazione di:

uno strumento giuridico vincolante (convenzione) sulla protezione dei whistleblower sulla base della Raccomandazione del Comitato dei Ministri $\mathrm{CM} / \operatorname{Rec}(2014) 7$, prendendo in considerazione i recenti sviluppi. ${ }^{12}$

9 Guseva c. Bulgaria, $\mathbb{\$} 37$. Vedi inoltre Shapovalov c. Ucraina, $\mathbb{} 68$ e Dammann c. Svizzera, $\mathbb{S} 52$.

10 Guja c. Moldavia.

11 Comitato dei Ministri del Consiglio d'Europa (2014), Raccomandazione CM/ $\operatorname{Rec}(2014) 7$ sulla protezione dei "whistleblower".

12 La risoluzione sottolinea la necessità di garantire la protezione accordata ai whistleblower anche agli impiegati della sicurezza nazionale o dei servizi di intelligence e alle aziende private che lavorano in questo campo nonché di garantire asilo ai whistleblower le cui rivelazioni soddisfano gli standard richiesti dal Consiglio d'Europa in tutti gli Stati membri. All'interno della stessa risoluzione del 23 giugno 2015, l'Assemblea parlamentare richiese agli Stati Uniti d'America "di permettere al Sig. Edward Snowden di ritornare in patria senza il timore di subire 
La protezione diretta dei whistleblower in base all'art. 10 CEDU è complementare alla severa e raffinata casistica sulla protezione delle fonti giornalistiche, garantendo un elevato livello di protezione per coloro che agiscono in funzione di fonti (confidenziali) per i giornalisti. Il diritto dei giornalisti di difendere le loro fonti è stato sostenuto in molti casi e dimostra la necessità di una protezione per i whistleblower, come spiegato nei casi della Corte Goodwin c. Regno Unito, Roemen e Schmit c. Lussemburgo, Voskuil c. Paesi Bassi, Tillack c. Belgio, Financial Times Ltd. C. Regno Unito e Nagla c. Lettonia. ${ }^{13}$

Nel presente capitolo, il riconoscimento di un diritto di accesso ai pubblici documenti e il diritto degli impiegati pubblici e dei dipendenti del settore privato di agire come whistleblower e fonti giornalistiche sarà inquadrato nel contesto giuridico del Consiglio d'Europa e dell'interpretazione della CEDU. Lo sviluppo e le caratteristiche di tali diritti, così come le loro limitazioni, verranno illustrati con riferimento alle sentenze di riferimento pronunciate della Corte, applicando l'art. 10 della CEDU a circostanze concrete, così come in relazione a documenti di policy rilevanti dell'Assemblea parlamentare e del Comitato dei Ministri del Consiglio d'Europa. L'applicazione di entrambe le "estensioni" della protezione ex art. 10 CEDU è rilevante specialmente in supporto ad un giornalismo d'indagine e indipendente nonché per i media e le organizzazioni non-governative (ONG) nell'esercizio del loro ruolo di pubblici guardiani in società democratiche trasparenti e sostenibili. Una straordinaria e importante caratteristica dell'estensione dell'ambito di applicazione dell'art. 10 della CEDU consiste nel fatto che sia il diritto di accesso ai pubblici documenti che la protezione dei whistleblower sono validi e applicabili nel campo della sicurezza nazionale e dei servizi segreti, campi tradizionalmente esclusi, secondo la normativa nazionale, dal campo della trasparenza in quanto viene data priorità alla segretezza e alla confidenzialità degli stessi.

\section{L'ARTICOLO 10 DELLA CEDU}

Il diritto alla libertà di espressione e di informazione è garantito dall'art. 10 della CEDU in tutti gli Stati membri del Consiglio d'Europa, dalla Norve-

persecuzioni in condizioni che non gli consentirebbero di richiamare la difesa dell'interesse pubblico".

13 Goodwin c. Regno Unito; Roemen e Schmit c. Lussemburgo; Voskuil c. Olanda; Tillack c. Belgio; Financial Times Ltd. ed altri c. Regno Unito e Nagla c. Lettonia. 
gia a Cipro, dall'Islanda all'Azerbaigian e dal Portogallo alla Russia. La tendenza verso una migliore garanzia di tale diritto è stata indubbiamente influenzata (nella maggior parte dei) Paesi membri del Consiglio d'Europa dall'applicazione dinamica dell'art. 10 della CEDU da parte della Corte. L'art 10 della CEDU afferma che:

1. Ogni persona ha diritto alla libertà d'espressione. Tale diritto include la libertà d'opinione e la libertà di ricevere o di comunicare informazioni o idee senza che vi possa essere ingerenza da parte delle autorità pubbliche e senza limiti di frontiera. Il presente articolo non impedisce agli Stati di sottoporre ad un regime di autorizzazione le imprese di radiodiffusione, cinematografiche o televisive.

2. L'esercizio di queste libertà, poiché comporta doveri e responsabilità, può essere sottoposto alle formalità, condizioni, restrizioni o sanzioni che sono previste dalla legge e che costituiscono misure necessarie, in una società democratica, alla sicurezza nazionale, all'integrità territoriale o alla pubblica sicurezza, alla difesa dell'ordine ed alla prevenzione dei reati, alla protezione della salute o della morale, alla protezione della reputazione o dei diritti altrui, per impedire la divulgazione di informazioni riservate o per garantire l'autorità e l'imparzialità del potere giudiziario.

L'art. 10(1) della CEDU contiene il principio del diritto alla libertà di espressione e di informazione, mentre l'art. 10(2) della CEDU apre, in relazione ai "doveri ed alle responsabilità" che vanno di pari passo all'esercizio di tale diritto, alla possibilità, per le pubbliche autorità, di interferire per mezzo di formalità, condizioni, restrizioni e addirittura sanzioni. In ogni modo, l'art. 10(2) riduce, al contempo, sostanzialmente la possibilità di interferire con la libertà di esprimere, ricevere e divulgare informazioni ed idee. L'interferenza da parte delle pubbliche autorità è consentita soltanto sotto la severa condizione che qualsiasi restrizione o sanzione debba essere "prevista dalla legge", ${ }^{14}$ debba perseguire uno "scopo legittimo" e, in ultima istanza e soprattutto, essere "necessaria in una società democratica".

La casistica della Corte dimostra che le proprie decisioni hanno consentito di conferire valore alla protezione della libertà di espressione, la libertà giornalistica, la libertà dei media ed il pubblico dibattito nei Paesi membri

14 Solo in pochi casi la Corte giunse alla conclusione che la condizone imposta dalla formula "prescritto dalla legge", la quale include la prevedibilità, la precisione e il grado di diffusione pubblica o l'accessibilità e che implica un minimo grado di protezione contro l'arbitrarietà, non fosse soddisfatta; per esempio nei casi Abmet Yıldırım c. Turchia; Youth Initiative for Human Rights c. Serbia e Guseva c. Bulgaria. 
della CEDU. In quasi 600 casi, la Corte ha ravvisato violazioni del diritto alla libertà di espressione e di informazione garantito dall'art. 10 della CEDU, sviluppando perciò un livello maggiore di protezione in confronto a quello previsto dalla normativa nazionale dello Stato convenuto. La giurisprudenza della Corte ha chiaramente ridotto le possibilità di interferire nei diritti alla libertà di espressione e di informazione evidenziando le caratteristiche di una società democratica in termini di tolleranza, apertura mentale, pluralismo e specialmente l'importanza della partecipazione al pubblico dibattito, inclusa la protezione di espressioni, idee e informazioni che "scandalizzano, offendono o creano disturbo".

La casistica della Corte ha riconosciuto la funzione preminente dei media e del giornalismo in un Paese sorretto dallo Stato di diritto, evidenziando, a cadenza regolare, che i media svolgono il ruolo vitale di pubblici guardiani e fornitori di informazioni in una democrazia. In ogni caso, le varie normative e i regolamenti nazionali continuano a ridurre la libertà di espressione, la ricerca di notizie e i contenuti mediatici. L'obiettivo di tali restrizioni consiste nel proteggere:

I. l'interesse nazionale (protezione della sicurezza dello Stato e dell'ordine pubblico);

II. la morale;

III. la reputazione o la privacy $\mathrm{o}$, in senso più generale, i diritti degli altri;

IV. la confidenzialità delle informazioni; $\mathrm{o}$

V. l'autorità e l'imparzialità della magistratura.

Altre previsioni legali mirano a proteggere i dati personali e proibiscono $\mathrm{i}$ "discorsi di odio" che incitano alla violenza, al razzismo, alla xenofobia, all'odio o alla discriminazione. Inoltre, le leggi sulle trasmissioni, le normative sui servizi dei media audiovisivi e le discipline giuridiche in materia di pubblicità o altre forme di "contenuti pubblicitari" contengono restrizioni sulla libertà di espressione o sui contenuti mediatici. ${ }^{15}$ In più occasioni, la Corte ha ripetuto che l'articolo 10 della CEDU non garantisce ai media la libertà di espressione senza alcuna restrizione, anche nel rispetto della divulgazione di tematiche di grande interesse pubblico.

Nel godere della protezione accordata loro dalla Convenzione, i giornalisti devono, nell'esercizio dei loro dovere, attenersi ai principi del giornalismo responsabile, ovvero agire in buona fede, fornire informazioni accurate ed affidabili, riportare in maniera oggettiva le opinioni

15 Per una visione d'insieme e un'analisi vedi Commissario per i diritti dell'uomo (2011) e Casadevall J. et al. (2012). 
di coloro che sono coinvolti in un pubblico dibattito ed astenersi da un puro scandalismo. ${ }^{16}$

Ciò significa anche che i media che applicano gli standard dell'etica giornalistica o i giornalisti che rispettano i principi del giornalismo responsabile sono fortemente protetti dall'art. 10 della CEDU. In ogni caso, ciò non comporta che i giornalisti debbano agire osservando le norme di buona pratica giornalistica in tutte le circostanze al fine di essere tutelati dall'art. 10 della CEDU. ${ }^{17}$ In alcuni casi, la Corte era dell'opinione che, nonostante fosse stato "consigliabile" per un quotidiano e per i propri giornalisti ottenere una presa di posizione in anticipo da parte di una persona criticata nelle pagine del giornale per il proprio coinvolgimento in un caso di frode e di uso improprio di fondi pubblici,

il mero fatto che costei non avesse compiuto il fatto non è sufficiente per ritenere che era giustificata l'interferenza con il diritto alla libertà di espressione della società ricorrente. ${ }^{18}$

\section{GOVERNI NAZIONALI NON POSSONO PIÜ DECIDERE IN MERITO AI LIMITI DELLA LIBERTÀ DEI MEDIA E DEI GIORNALISTI}

Fino a qualche decennio fa, i limiti e le restrizioni alla libertà di espressione venivano determinati dai governi nazionali e valutati, in ultima istanza, dalle loro autorità giudiziarie nazionali, senza alcun controllo esterno. Questo modello venne profondamente modificato in Europa con l'avvento della CEDU ed il proprio quadro di consolidamento, nel quale la Corte gioca in ruolo cruciale. ${ }^{19}$

A partire dalla sentenza della Corte Sunday Times c. Regno Unito (n. 1$)^{20}$ nel 1979, è diventato chiaro che l'art. 10 della CEDU ha effettivamente ridotto la sovranità nazionale e l'ambito delle limitazioni nazionali per re-

16 Armellini et al. c. Austria, $\$ 41$.

17 Flux e Samson c. Moldavia; Timpul Info-Magazin e Anghel c. Moldavia; e Standard Verlags GmbH c. Austria.

18 Krone Verlag GmbH \& Co c. Austria (n. 5). Vedi inoltre Standard Verlags GmbH c. Austria (n. 3).

19 Vedi inoltre Harris D. J. et al. (2009).

20 Sunday Times c. Regno Unito (n. 1). Qualche anno prima, nel suo primo giudizio sulla libertà d'espressione (Handyside c. Regno Unito), la Corte rimarcò l'importanza della libertà d'espressione in una società democratica, ma non rilevò alcuna violazione dell'art. 10 della Convenzione nel caso in parola, in quanto la protezione di minori venne considerata una giustificazione per l'interferenza delle pubbli- 
stringere le libertà di espressione e di informazione. In più occasione, la Corte ha stabilito un livello di protezione più elevato per i contributi giornalistici su temi di pubblico interesse, riconoscendo anche "il diritto del pubblico di essere informato in forma adeguata" su tematiche di interesse per la società. Nel corso degli anni, l'abbondanza dei casi affrontati dalla Corte ha reso chiaro che le normative nazionali che proibiscono, restringono o sanzionano le espressioni o le informazioni quali forme di pubbliche comunicazioni possono essere attuate soltanto se sono: (I) sufficientemente definite dal punto di vista legislativo; (II) non adottate in forma arbitraria; (III) giustificate da uno scopo legittimo; e, soprattutto, (IV) considerate necessarie in una società democratica. La Corte ha altresì stabilito in varie occasioni che la CEDU è uno "strumento vivente" e che, in quanto tale intende "garantire non diritti teorici o illusori, bensì diritti pratici ed effettivi". ${ }^{21}$

Con il caso Sunday Times come punto di partenza nel 1979, le normative di molti Paesi europei sono state ritenute lesive dell'art. 10 della CEDU dopo che giornalisti, caporedattori, case editrici, emittenti, accademici, politici, artisti, attivisti o ONG hanno lamentato dinanzi alla Corte di essere divenuti vittime di interferenze nella loro libertà di espressione illegittime, ingiustificabili o sproporzionate. Come conseguenza della casistica della Corte, ed a causa del carattere vincolante della CEDU, gli Stati membri sono tenuti a modificare ed a rafforzare i loro strumenti di protezione della libertà di espressione ai sensi dell'art. 1 della CEDU. Questo approccio ha riguardato, in particolare, la protezione dei contributi giornalistici, del dibattito politico e della discussione di questione di pubblico interesse, rigettando alcune delle limitazioni tradizionali alla libertà di espressione in molti Paesi che non possono più essere considerate giustificate in una società democratica. In anni più recenti, la Corte ha anche garantito l'accesso ai pubblici documenti facendolo ricadere nell'ambito di applicazione della CEDU e, in diverse occasioni, ha ritenuto che le sanzioni imposte contro i whistleblower per aver rivelato informazioni di pubblico interesse abbiano leso il loro diritto alla libertà di espressione e di informazione (cfr. infra).

Allo stesso tempo, la Corte è diventata un attore importante nella difesa della libertà di stampa contro nuove iniziative o tentativi di restringerla. La giurisprudenza della Corte si è fortemente opposta all'introduzione di nuovi limiti o obblighi aggiuntivi che rischiano di danneggiare l'importante ruolo dei media critici ed indipendenti in una società democratica. Un

che autorità nella pubblicazione "Little Red Schoolbook" e nei confronti del suo editore, il sig. Handyside.

21 Vedi, per esempio, Centro Europa 7 S.R.L. e Di Stefano c. Italia. 
esempio eloquente ne è la sentenza della Corte Mosley c. Regno Unito del 2001. La Corte ha ritenuto che il diritto alla privacy garantito dall'art. 8 della CEDU non richiede che i media debbano preventivamente dare notizia della pubblicazione dei contenuti alle persone citate in essi. ${ }^{22} \mathrm{Nel}$ caso Wegrzynowsky e Smolczewski c. Polonia, la Corte ha fornito una sentenza interessante riguardo alla richiesta di rimuovere un articolo pubblicato online che due legali consideravano lesivo della loro reputazione. Nei precedenti processi di diffamazione dinanzi alle corti polacche, la richiesta era stata ritenuta fondata su informazioni insufficienti e l'articolo continuò a rimanere accessibile al pubblico sul sito internet del quotidiano. La Corte ha ritenuto che il giornale non era obbligato a rimuovere l'articolo dal proprio archivio internet. Ha accettato che la Polonia avesse adempiuto il proprio obbligo di bilanciare, da un lato, i diritti garantiti dall'art. 10 della CEDU e, dall'altro lato, dall'art. 8 della CEDU. La Corte ritiene che la rimozione dell'articolo online per tutelare la reputazione del ricorrente sarebbe stata, nelle circostanze del caso, sproporzionata in base all'art. 10 della CEDU e che una rettifica o un commento aggiunto sul sito avrebbe costituito un rimedio sufficiente ed adeguato. ${ }^{23}$

La Corte ha anche rafforzato il diritto dei singoli di accedere a internet in una sentenza contro un blocco totale di contenuti digitali, nella quale ha asserito che internet è diventato, nel frattempo, uno dei principali mezzi per l'esercizio del diritto alla libertà di espressione e di informazione. La Corte ha chiarito che la restrizione dell'accesso a una fonte di informazioni è compatibile con la CEDU soltanto se sussiste un quadro giuridico severo che contenga garanzie. La sentenza ha altresì precisato che le corti nazionali avrebbero dovuto comprendere che una tale misura avrebbe reso inaccessibile un grande quantitativo di informazioni colpendo, di conseguenza, $\mathrm{i}$ diritti degli utenti internet, con un importante effetto collaterale sul loro diritto di accedere ad internet. ${ }^{24}$

\section{Mosley c. Regno Unito.}

23 Wegrzynowski e Smolczewski c. Polionia. Confronta con Corte di giustizia del'Unione europea, Grande Camera 13 maggio 2013, C 131/12, Google Spain.

24 Abmet Yildirn c. Turchia. Anche nel caso Del AS c. Estonia, la Corte statuì su un importante problema relativo alla libertà d'espressione su internet, più precisamente sulla responsabilità (limitata) dei gestori di siti di notizie online in relazione ai commenti postati dagli utenti contenenti insulti e diffamazioni. In questo caso, aver sanzionato colui che ha pubblicato il sito internet in quanto responsabile di contenuto diffamatorio postato dagli utenti non determinò una violazione dell'art.10. Questo approccio fu confermato dal giudizio della Grande Camera in Del AS del 16 giugno 2015. Mentre la Corte riconobbe che, per quanto riguarda l'esercizio della libertà di espressione, possono derivare importanti benefici da in- 
È importante notare che la casistica della Corte ha statuito che le autorità nazionali non dovrebbero soltanto astenersi dalle interferenze nella libertà di espressione e nella libertà di stampa non necessarie in una società democratica, ma sono altresì soggette agli obblighi positivi di proteggere il diritto alla libertà di espressione contro interferenze da parte di individui privati od organizzazioni. In un caso contro la Svezia la Corte ha chiarito che, nonostante il proprio compito non fosse quello di chiarire contenziosi di natura puramente privata:

essa non può mantenere un atteggiamento passivo quando l'interpretazione di un atto normativo, che si tratti di una disposizione testamentaria, di un contratto tra privati, di un documento pubblico, di una previsione statutaria o di un provvedimento amministrativo, da parte di una corte nazionale appaia irragionevole, arbitraria, discriminatoria $\mathrm{o}$, in senso più lato, in disaccordo con i principi sottesi alla Convenzione. ${ }^{25}$

La Corte ha altresì evidenziato che:

in aggiunta all'obbligo primario negativo di uno Stato di astenersi dall'interferire nelle garanzie della Convenzione, possono esistere obblighi positivi inerenti a tali garanzie. La responsabilità di uno Stato potrebbe dunque essere ravvisata come conseguenza del mancato rispetto di tali obblighi. ${ }^{26}$

Nella sentenza Özgür Gündem c. Turchia, la Corte ha sviluppato questo approccio ritendo che:

l'esercizio reale ed effettivo della libertà di espressione non dipende solamente dal dovere, da parte dello Stato di non interferire, ma può anche richiedere misure positive di protezione, anche nella sfera delle relazioni tra individui.

In seguito ad una serie di omicidi, sparizioni, attacchi, persecuzioni, sequestri e confische, il quotidiano Özgür Gündem ha cessato le proprie pubblicazioni. Secondo la Corte, le autorità turche non erano state in grado di

ternet, affermò altresì di essere altrettanto consapevole che la possibilità per le persone lese da dichiarazioni diffamatorie o da altri tipi di contenuto illecito di intentare un'azione di responsabilità che possa costituire un rimedio effettivo contro le violazioni dei diritti della personalità debba essere mantenuta” ( $(110)$. Per un commento critico, vedi Voorhoof D. (2015c).

25 Khurshid Mustafa e Tarzibachic. Svezia.

26 Fuentes Bobo c. Spagna; Özgür Gündem c. Turchia; VgT Verein gegen Tierfabriken c. Svizzera (n. 1); VgT Verein gegen Tierfabriken c. Svizzera (n. 2); e Wojtas-Kaleta c. Polonia. Vedi inoltre Appleby ed altri c. Regno Unito. 
adempiere al loro obbligo positivo di proteggere il giornale ed i suoi giornalisti nell'esercizio della loro libertà di espressione. ${ }^{27}$

In altri casi, la Corte ha altresì adottato la teoria degli obblighi positivi applicando altre previsioni della CEDU, per esempio in casi di omicidi di giornalisti che configuravano non soltanto la violazione dell'art. 10, bensì anche del diritto alla vita (art. 2) o del divieto di tortura o di trattamenti disumani o degradanti (art. 3), in combinazione con il diritto a un ricorso effettivo (art. 13). ${ }^{28}$ In un caso recente relativo ad un violento attacco contro un giornalista, la Corte ha ripetuto che gli Stati membri sono tenuti, in base agli obblighi positivi sanciti dalla CEDU, a creare un ambiente favorevole per la partecipazione al pubblico dibattito da parte di tutte le persone interessate, consentendo loro di esprimere le loro opinioni e idee senza timore. In questo particolare caso, l'incapacità di svolgere un'indagine efficace portò la Corte a ritenere che l'investigazione avvenuta dopo che un giornalista aveva lamentato un maltrattamento fosse stata inefficace e che vi fosse una violazione dell'art. 3 della CEDU (divieto di tortura o trattamenti inumani o degradanti). In tale particolare caso, un giornalista era stato vittima di un violento attacco da parte di due uomini soltanto poche ore dopo aver pubblicato un articolo in cui aveva accusato un alto ufficiale dell'esercito di corruzione ed attività illegali. Il giornalista venne colpito più volte con un oggetto contundente e venne anche preso a pugni dai propri aggressori. L'attacco avvenne al di fuori degli uffici del giornale. Nonostante fosse stata avviata una formale investigazione penale in relazione all'attac$\mathrm{co}$, non si intrapresero ulteriori passi per identificare gli autori. In relazione all'art. 3 della CEDU, il giornalista sostenne che ci fossero agenti governativi dietro l'attacco contro di lui e che la polizia non era stata in grado di portare avanti un'investigazione effettiva del maltrattamento. La Corte ravvisò vari difetti nell'investigazione poliziesca, i quali la spinsero a ritenere che l'investigazione del caso era stata inefficace e che era stato, di conseguenza, leso l'art. 3 della CEDU. ${ }^{29}$ 


\section{MEDIA, I GIORNALISTI, LE ONG E LA SOCIETÀ CIVILE QUALI PUBBLICI GUARDIANI}

La Corte ha chiarito che, in una società democratica, oltre ai media, le ONG, i gruppi di promozione e le organizzazioni che trasmettono un messaggio al di fuori dell'opinione tradizionale debbano essere messi in grado di svolgere le loro attività in maniera effettiva e di poter fare affidamento ad un elevato livello di libertà di espressione, essendovi:

Un forte pubblico interesse nel consentire a tali gruppi e a individui che si pongono al di fuori dell'opinione tradizionale di contribuire al pubblico dibattito divulgando informazioni ed idee su questioni di interesse pubblico generali quali la salute e l'ambiente. ${ }^{30}$

In una società democratica, le pubbliche autorità dovrebbero essere sottoposte ad un'indagine continua da parte dei cittadini e ognuno deve essere in grado di porre l'attenzione pubblica su situazioni ritenute contrarie alla legge. ${ }^{31}$

Particolare attenzione è posta al pubblico interesse coinvolto nella rivelazione di informazioni che contribuiscono al dibattito su materie di pubblico interesse:

In un sistema democratico gli atti o le omissioni del governo devono essere soggetti ad una severa indagine, non soltanto da parte delle autorità legislative e giudiziarie, ma anche da parte dei media e dell'opinione pubblica. L'interesse che il pubblico può avere nei confronti di particolari informazioni può talvolta essere talmente forte da avere la precedenza anche su un obbligo di riservatezza imposto per legge. ${ }^{32}$

In tali circostanze, i giornalisti non dovrebbero essere perseguiti o sanzionati per aver infranto la confidenzialità o per aver utilizzato documenti ot-

30 Steel ed altri c. Regno Unito. Vedi inoltre Hertel c. Svizzera; VgT Verein gegen Tierfabriken c. Svizzera (n. 1); VgT Verein gegen Tierfabriken c. Svizzera (n. 2); Vides Aizsardzibas Klubs c. Lettonia e Mamère c. Francia. Vedi inoltre Open Door e Dublin Well Women c. Irlanda; Hashman e Harrup c. Regno Unito; Çetin e Şakar c. Turchia; Women on Waves c. Portogallo; Hyde Park ed altri c. Moldavia (n. 5-6); Schwabe e M.G. c. Germania; Tatár e Fáber c. Ungheria; Kudrevičius ed altri c. Lituania (relativo alla Grande Camera) e Taranenko c. Russia.

31 Vides Aizsardzibas Klubs c. Lettonia. Vedi anche Tatár e Fáber c. Ungheria.

32 Guja c. Moldavia e Bucur e Toma c. Romania. Vedi Morice c. Francia. 
tenuti illegalmente. ${ }^{33}$ La Corte ha accettato che l'interesse sotteso alla pubblicazione delle informazioni derivanti da una fonte che ha ottenuto e ritrasmesso le informazioni in maniera illegittima possa in certe circostanze essere più importante di quello di conservare, per un privato o un'entità pubblica, la riservatezza delle informazioni. Un giornale che ha pubblicato uno scambio di e-mail tra due personaggi pubblici ottenuto illegalmente, direttamente riferito alla discussione su una questione di serio interesse pubblico, può essere tutelato, in base all'art. 10 della CEDU, dalle richieste basate sul diritto alla privacy protetto dall'art. 8 della CEDU. ${ }^{34}$

In un caso che riguardava la condanna di quattro giornalisti per aver registrato e trasmesso un'intervista utilizzando telecamere nascoste, la Corte ha ritenuto che le autorità svizzere avessero violato i diritti dei giornalisti $e x$ art. 10 della CEDU. La Corte ha evidenziato che l'utilizzo di telecamere nascoste da parte dei giornalisti era finalizzato a fornire informazioni di pubblica natura su un argomento di interesse generale, pertanto la persona filmata non venne ripresa come persona, ma come broker professionista. La Corte ha ritenuto che l'interferenza nella vita privata del broker non era stata sufficientemente forte da sovrastare il pubblico interesse ad ottenere informazioni che denunciassero le cattive pratiche nel campo del brokeraggio assicurativo. ${ }^{35}$

Nella sentenza della Grande Camera Stoll c. Svizzera, la Corte ha confermato che la libertà di stampa assume un'importanza addirittura maggiora in circostanze in cui le attività e le decisioni dello Stato sfuggono ad un'in-

33 Fressoz e Roire c. Francia; Dammann c. Svizzera; Dupuis ed altri c. Francia; Peev c. Bulgaria e Guja c. Moldavia. Vedi inoltre Radio Twist c. Slovacchia e Pinto Coelho c. Portogallo.

34 Jonina Benediktsdottir c. Islanda; Fressoz e Roire c. Francia e Radio Twist c. Slovacchia.

35 Haldimann ed altri c. Svizzera. Confrontato con Tierbefreier E.V. c. Germania. In questo caso la Corte europea prese in considerazione che un'ingiunzione contro l'uso da parte di organizzazioni per i diritti degli animali di video ripresi illegalmente da giornalisti non costituiva nessun tipo di violazione dell'art. 10 della CEDU, in quanto l'ingiunzione non includeva l'utilizzo a scopo giornalistico da parte dei media, ma solamente l'utilizzo ingiustificato da parte delle associazioni per i diritti degli animali comprendente il video del film "Poisoning for profit" sul proprio sito, accusando un'impresa di aver infranto la regolamentazione relativa al trattamento degli animali. La Corte osservò che i tribunali locali avevano esaminato attentamente se accogliere l'ingiunzione in questione violasse in qualche modo il diritto di libertà d'espressione dell'associazione sopracitata, riconoscendo pienamente l'impatto del diritto di libertà d'espressione in un dibattito su un tema di interesse pubblico. La Corte sottolinò la mancanza di evidenze nonostante le accuse mosse nei confronti del film "Poisoning for profit" fossero corrette, secondo cui la compagnia C. infrangeva sistematicamente le leggi. 
dagine democratica o giudiziaria sulla base della loro natura confidenziale o segreta. Il processo e la condanna di un giornalista per aver rivelato informazioni considerate confidenziali o segrete potrebbe scoraggiare coloro che operano nel settore mediatico dall'informare il pubblico su materie di pubblico interesse. Di conseguenza, la stampa potrebbe non essere più in grado di svolgere il proprio ruolo vitale di pubblico guardiano e l'abilità della stampa di fornire informazioni accurate ed affidabili risulterebbe compromessa. ${ }^{36}$ In casi in cui i giornalisti riportano informazioni confidenziali in modo scandalistico ${ }^{37} \mathrm{o}$ in cui i documenti divulgati non contribuiscono al pubblico dibattito in maniera concreta o effettiva o riguardano soltanto informazioni circa la vita privata della persona coinvolta, ${ }^{38}$ la Corte ha accettato un'interferenza (proporzionata) nella loro libertà di espressione.

Nei casi in cui i giornalisti o i media non sono stati in grado di fornire prove affidabili o rilevanti per le loro (gravi) asserzioni, insinuazioni o accuse, la Corte accetta le condanne e le sanzioni (proporzionate) imposte dalle autorità nazionali in quanto non lesive dell'art. 10 della CEDU. ${ }^{39} \mathrm{La}$ necessità che un giornalista debba provare che le accuse formulate in un articolo fossero "sostanzialmente vere" bilanciando le probabilità costituisce una restrizione giustificata del diritto della libertà di espressione ai sensi dell'art. 10(2) della CEDU. ${ }^{40}$ In alcuni casi l'ovvia mancanza di prove a sostegno delle accuse pubblicati ha portato la Corte a definire addirittura inammissibile un ricorso $e x$ art. 10 CEDU..$^{41}$ Dall'altro lato, la Corte ha altresì preso in considerazione che i reportage mediatici su "racconti" o "voci"

36 Stoll c. Svizzera. Vedi inoltre Goodwin c. Regno Unito e Fressoz e Roire c. Francia.

37 Stoll c. Svizzera. Vedi inoltre Armellini ed altri c. Austria, $\mathbb{\$} 41$.

38 Leempoel e S.A. Ciné Revue c. Belgio e Marin c. Romania. Vedi inoltre De Diego Nafria c. Spagna e Cumpănă e Mazăre c. Romania. Vedi inoltre Ruusunen c. Finlandia e Ojala e Etukeno Oy c. Finlandia.

39 Prager e Oberschlick c. Austria; McVicar c. Regno Unito; Perna c. Italia; Radio France c. Francia; Chauvy c. Francia; Pedersen e Baadsgaard c. Danimarca; Rumyana Ivanova c. Bulgaria; Alithia Publishing Company Ltd. e Constantinides c. Cipro; Backes c. Cipro; Flux c. Moldavia (n. 6); Cuc Pascu c. Romania; Petrina c. Romania; Brunet-Lecomte ed altri c. Francia; Kania e Kittel c. Polonia; Ziembinski c. Polonia; Růžový panter, o.s. c. Repubblica Ceca; Novaya Gazeta e Borodyanskiy c. Russia; Lavric c. Romania; Salumäki c. Finlandia e Armellini ed altri c. Austria. In alcuni casi la Corte non riscontrò violazioni dell'art. 10 , mentre affermò che ai richiedenti non venne garantito un processo giusto e che vi fu una violazione dell'art. 6(1) della Convenzione: vedi, per esempio, Constantinescu c. Romania e Mihaiu c. Romania.

40 McVicar c. Regno Unito e Pedersen e Baadsgaard c. Danimarca.

41 Vedi, per esempo, Lászl Keller c. Ungheria; Corneliu Vadim Tudor c. Romania; Falter Zeitschriften GmbH c. Austria; Tomasz Wolek, Rafal Kaspr w e Jacek Lęsi c. Polonia e 
diffuse da persone diverse dal ricorrente o "pubbliche opinioni" debbano essere protette come parte del ruolo di "pubblici guardiani" svolto dai media. ${ }^{42}$ In più occasioni la Corte ha accettato che giudizi, asserzioni o esternazioni abbiano "una base fattuale solo ridotta" o che fosse sufficiente che non ci fosse "alcuna prova che la descrizione di fatti fornita nell'articolo fosse completamente falsa", o che le "opinioni si basassero su fatti che non sono stati dimostrati falsi". ${ }^{43}$ I giudizi di valore e le critiche possono basarsi su "asserzioni non confermate o voci". ${ }^{44}$ La Corte non accetta il ragionamento delle corti nazionali secondo cui le accuse di colpe gravi formulate contro individui o personalità pubbliche debbano preventivamente essere provate in processi penali. ${ }^{45} \mathrm{Nel}$ caso Kasabova la Corte ha spiegato che:

Mentre una condanna definitiva corrisponde, di principio, all'inconfutabile prova che una persona abbia commesso un reato, ridurre a ciò il modo per provare le accuse di una condotta criminosa nel contesto di un caso di diffamazione è chiaramente irragionevole, anche se deve essere tenuta in considerazione, come richiesto dall'art. 6(2), la presunzione di innocenza di una persona. ${ }^{46}$

Definire un atto o un comportamento di un politico "illegale" è da considerarsi come l'espressione di un'opinione giuridica personale che corrisponde ad un giudizio di valore del quale non si può chiedere di provarne l'esattezza. ${ }^{47}$

Le leggi sulla diffamazione e i relativi processi penali non possono essere giustificati se il loro scopo o il loro effetto consistono nel prevenire una critica legittima nei confronti dei pubblici ufficiali o lo smascheramento di

Vittorio Sgarbi c. Italia. Vedi inoltre Verdens Gang e Kari Aarsted Aase c. Norvegia; Gaudio c. Italia; Dunca e SC Nord Vest Press SRL c. Romania e Ciuvică c. Romania.

42 Vedi, per esempio, Thorgeir Thorgeirson c. Islanda e Cihan Özturk c. Turchia.

43 Vedi, per esempio, Nilsen e Johnsen c. Norvegia; Dalban c. Romania; Dichand ed altri c. Austria e Flux e Samson c. Moldavia.

44 Timpul Info-Magazin e Anghel c. Moldavia. Vedi inoltre Cihan Özturk c. Turchia. La Corte ritenne comunque che, avendo le autorità già avviato procedimenti penali contro il richiedente per violazione dei doveri, "vi era una base fattuale sufficiente affichè il richiedente sviluppasse in'analisi critica della situazione e promuovesse domande relative al progetto di ripristino".

45 Vedi Nilsen e Johnsen c. Norvegia; Flux v. Moldavia (n. 6); Folea c. Romania; Dyundin c. Russia; Godlevskiy c. Russia e Kydonis c. Grecia. Confronta con Constantinescu c. Romania e Petrina c. Romania. Vedi inoltre Brosa c. Germania e Erla Hlynsottir c. Islanda (n. 3).

46 Kasabova c. Bulgaria.

47 Vides Aizsardzibas Klubs c. Lettonia. Vedi inoltre Selistö c. Finlandia e Karhuvaara e Iltalehti c. Finlandia. Vedi Brosa c. Germania. 
illeciti ufficiali o episodi di corruzione. Di un diritto di citare in giudizio un soggetto per aver diffamato la reputazione di un pubblico ufficiale si può facilmente abusare. Esso potrebbe impedire un dibattito libero e aperto su questioni di pubblico interesse o valutazioni sulla spesa di denaro pubblico. ${ }^{48}$ Specialmente in casi in cui vengono pubblicate informazioni su presunti episodi di corruzione, frode o attività illecite in cui sono coinvolti politici, funzionari pubblici o istituzioni pubbliche, i giornalisti, gli editori, i media e le ONG dovrebbero essere in grado di fare affidamento ai più elevati standard di protezione della libertà di espressione. La Corte ha evidenziato che:

in un Paese democratico in cui vige lo Stato di diritto, l'uso di metodi impropri da parte di pubbliche autorità è esattamente il tipo di argomenti sui quali il pubblico ha il diritto di essere informato. ${ }^{49}$

La Corte ritiene che:

la stampa è uno dei mezzi tramite il quale i politici e l'opinione pubblica possono verificare che il denaro pubblico venga speso secondo $i$ principi contabili e non per arricchire determinati individui. ${ }^{50}$

In alcuni casi, la Corte ha ordinato al governo dello Stato membro convenuto di assumere misure concrete ed urgenti al fine di rispettare e ripristinare immediatamente la libertà di espressione e di opinione del ricorrente, come, per esempio, nel caso Fatullayev c. Azerbaigian (nel quale la Corte ha ordinato l'immediato rilascio dal carcere di un giornalista condannato per aver calunniato il governo) o nel caso Youth Initiative for Human Rights c. Serbia (in cui la Corte ha ordinato ai servizi segreti serbi di fornire le informazioni richieste dalla ONG ricorrente). ${ }^{51}$

Le interferenze da parte delle pubbliche autorità in via processuale o altre misure giudiziarie relative alla ricerca giornalistica o altre attività investigative o di raccolta di notizie dovrebbero essere sottoposte ad una valutazione scrupolosa in prospettiva dell'art. 10 della CEDU. ${ }^{52}$ In un caso attualmente pendente, alla Grande Camera della Corte è stato chiesto di valutare

48 Cihan Özturk c. Turkey.

49 Voskuil c. Olanda.

50 Krone Verlag GmbH \& Co c. Austria (n. 5).

51 Fatullayev c. Azerbaigian e Youth Initiative for Human Rights c. Serbia.

52 Vedi De Haes e Gijsels c. Belgio; Fressoz e Roire c. Francia; Bladet Tromsø e Stensaas c. Norvegia; Du Roy e Malaurie c. Francia; Thoma c. Lussemburgo; Colombani ed altri c. Francia; Vides Aizsardzibas Klubs c. Lettonia; Radio Twist c. Slovacchia; Ukrainian Media Group c. Ucraina e Dupuis ed altri c. Francia. Vedi anche Nagla c. Lettonia. 
se l'arresto e la condanna di un fotografo della stampa finlandese per aver disobbedito alla polizia mentre stava riportando una manifestazione sfociata in violenze configurasse la lesione della libertà di espressione dello stesso ai sensi dell'art. 10 della CEDU. Il ricorrente del caso, il sig. Pentikäinen, è un fotografo e giornalista che stava immortalando alcuni attimi di un'ampia manifestazione a Helsinki. L'evento sfociò in una sommossa e la polizia decise di isolare l'area. Contravvenendo all'ordine imposto dalla polizia, un gruppo di circa venti persone rimase nell'area, incluso Pentikäinen, il quale ritenne che l'ordine di sgomberare l'area fosse rivolto soltanto ai manifestanti e non a lui stesso, in quanto egli stava lavorando in qualità di giornalista. Tentò anche di far capire alla polizia che egli era un rappresentante dei media mostrando il proprio cartellino. Poco tempo dopo venne arrestato dalla polizia insieme ai manifestanti. Venne tenuto in custodia da parte della polizia per 17 ore e poi accusato dal pubblico ministero di aver disobbedito alla polizia. La corte finlandese lo giudicò colpevole di tale delitto, ma non fu imposta alcuna sanzione in quanto la corte ritenne che le sue azioni fossero scusabili. Nel proprio ricorso alla Corte di Strasburgo, Pentikäinen lamentò che, con l'arresto e la condanna, erano stati lesi i propri diritti ai sensi dell'art. 10 della CEDU, essendogli stato impedito di svolgere il proprio mestiere di giornalista mentre stava raccogliendo notizie di pubblico interesse. La Corte ha riconosciuto che Pentikäinen era stato oggetto, nella propria qualità di fotografo di stampa e giornalista, di un'interferenza nella propria libertà di espressione. Tuttavia, poiché l'interferenza era prevista dalla legge, perseguiva uno scopo legittimo (la protezione dell'incolumità pubblica e la prevenzione di disordini e reati) ed era da considerarsi necessaria in una società democratica, non vi era stata alcuna violazione dei diritti $e x$ art. 10 della CEDU. La Corte ha altresì ritenuto che il fatto che il ricorrente fosse un giornalista non gli conferiva un più ampio diritto di rimanere sul luogo dei manifestanti e che il comportamento per il quale era stato ritenuto colpevole non riguardava l'attività giornalistica in quanto tale, bensì il suo rifiuto di eseguire un ordine impartito dalla polizia quando la manifestazione era sfociata in una sommossa. Con una decisione presa a maggioranza (cinque voti contro due) la Corte concluse, di conseguenza, che la corte finlandese aveva ben bilanciato gli interessi in gioco nel caso e che non vi era stata alcuna violazione dell'art. 10 della CEDU. Pertikäinen richiese, sostenuto dall'Unione dei giornalisti finlandesi, dalla Federazione internazionale dei giornalisti e dalla Federazione europea dei giornalisti, un deferimento alla Grande Camera, argomentando che la decisione della Corte rischiava di affievolire la libertà di stampa e i diritti dei giornalisti che trattano argomenti rilevanti per la società. Il 2 giugno 2014 il collegio giudicante decise di deferire il Caso alla 
Grande Camera, ove l'udienza si tenne il 17 dicembre 2014. La sentenza finale è prevista per la seconda metà del 2015. ${ }^{53}$ La sentenza della Grande Camera nel caso Pentikäinen c. Finlandia avrà senza dubbio un impatto enorme sui ricorsi futuri riguardanti il diritto, per i giornalisti, di ottenere notizie in zone di conflitto, manifestazioni o proteste violente, in cui sono coinvolte le forze armate o la polizia. Qualora fosse confermata la sentenza della Corte, secondo cui non cui fu, nel presente caso, alcuna violazione dell'art. 10 della CEDU, tale caso potrebbe sortire un "effetto dissuasivo" sulla libertà di stampa ${ }^{54}$.

\section{VERSO UN DIRITTO DI ACCESSO AI DOCUMENTI UFFICIALI}

Uno sviluppo importante per l'espansione del diritto alla libertà di espressione e di informazione si trova nella casistica della Corte relativa all'accesso ai pubblici documenti. A lungo la Corte si è rifiutata di applicare l'art. 10 della CEDU in casi di diniego di accesso ai pubblici documenti. ${ }^{55} \mathrm{Nei}$ casi Leander c. Svezia, Gaskin c. Regno Unito e Guerra e altri c. Italia la Corte ha evidenziato che:

il diritto di ottenere informazioni ... vieta, in sostanza, ad un governo di impedire ad una persona di ottenere informazioni che altri vorrebbero o che potrebbero voler fornirgli. Tale libertà non può essere intesa come imposizione nei confronti di uno Stato, in circostanze come nel presente caso, di obblighi positivi di raccogliere e divulgare esso stesso informazioni. ${ }^{56}$

Nel caso Roche c. Regno Unito del 2005 la Grande Camera ha fatto riferimento alle sentenze Leander, Gaskin e Guerra non ravvisando alcuna ragione "per non applicare tale giurisprudenza consolidata". ${ }^{57}$

Questo approccio della Corte contrastava fortemente con l'interpretazione data dalla Corte interamericana dei diritti umani nella propria sen-

53 Pentikäinen c. Finlandia. Vedi Voorhoof D. (2014b).

54 Nella sentenza Pentikäinen c. Finlandia (Grande Camera), Ricorso n. 11882/10, 20 ottobre 2015, la Corte non ha poi rilevato una violazione dell'art. 10 della CEDU (nota del traduttore).

55 La Corte seguì un percorso nuovo in Sdruženi Jihočeské Matky c. Repubblica Ceca. Vedi anche Hins e

Voorhoof (2007).

56 Leander c. Svezia, $\mathbb{\$} 74 ;$ Gaskin c. Regno Unito, $\$ 52$ e Guerra ed altri c. Italia, $\mathbb{} 53$.

57 Roche c. Regno Unito, SS 172-173. 
tenza del 19 settembre 2006 relativa al caso Claude Reyes e altri c. Cile. La Corte ha ritenuto all'unanimità che era stato leso il diritto alla libertà di espressione garantito dall'art. 13 della Convenzione americana sui diritti umani statuendo che tale diritto "tutela i diritti di tutti gli individui di richiedere accesso a informazioni detenute dallo Stato, con le eccezioni permesse dalle restrizioni contenute nella Convenzione". Curiosamente, la Corte interamericana dei diritti dell'uomo ha posto l'accento sul nesso tra il diritto di accesso alle informazioni detenute dallo Stato e la democrazia. ${ }^{58}$

L'approccio della Corte nel negare un diritto di accesso ali pubblici documenti in base all'art. 10 della CEDU contrastava anche con la Raccomandazione del 2002, la quale poneva l'accento sulla necessità di includere o incorporare nel diritto alla libertà di espressione il diritto di cercare informazioni e il diritto di accesso ai pubblici documenti. ${ }^{59}$

Ciononostante, la Corte ha sostenuto, in una sentenza del 2007, che:

devono sussistere ragioni particolarmente forti per qualsiasi misura che colpisce tale ruolo della stampa e limita l'accesso ad informazioni che il pubblico ha il diritto di ricevere ${ }^{60}$

ammettendo implicitamente almeno un diritto di accesso alle informazioni. Nella primavera del 2009, la Corte ha pronunciato due importanti sentenze nelle quali ha riconosciuto, entro determinati limiti, un diritto di accesso ai documenti ufficiali. La Corte ha specificato che, se autorità pubbliche detengono informazioni necessarie per un pubblico dibattito, il rifiuto di fornire documenti rilevanti a coloro che ne richiedono l'accesso configura una violazione del diritto alla libertà di espressione e di informazione come sancito dall'art. 10 della CEDU. Nel caso TASZ c. Ungheria la sentenza della Corte ha menzionato il "potere censorio di un monopolio dell'informazione" se autorità pubbliche si rifiutano di rivelare informazioni necessarie ai media o a organizzazioni della società civile per svolgere la loro funzione di pubblici guardiani. La Corte ha altresì ritenuto che lo Stato è

58 Corte interamericana dei diritti umani 19 settembre 2006, Claude Reyes ed altri c. Cile, www.corteidh.or.cr. Bisognerebbe tenere in considerazione che, a differenza dell'art. 10 della CEDU e similmente all'art. 19 del Patto internazionale sui diritti civili e politici, il diritto garantito dall'art.13 della Convenzione americana sui diritti umani comprende anche liberta "di ricercare" informazioni e idee separatamente dal diritto di divulgare e ricevere informazioni e idee.

59 Comitato dei Ministri del Consiglio d'Europa (2002), Raccomandazione $\operatorname{Rec}(2002) 2$.

60 Timpul Info-Magazin e Anghel c. Moldavia. 
tenuto a non impedire il flusso di informazioni richieste dai giornalisti o da cittadini interessati. La Corte ha fatto riferimento alla propria giurisprudenza in materia nella quale ha riconosciuto che il pubblico ha diritto di ricevere informazioni di interesse generale e che è richiesta una valutazione particolarmente attenta da parte della Corte per stabilire se le misure adottate da un governo nazionale possano scoraggiare la partecipazione della stampa, uno dei pubblici guardiani della società, al pubblico dibattito su tematiche di legittimo interesse pubblico, anche quando tali misure rendano l'accesso alle informazione meramente più complicato. La Corte ha evidenziato ancora una volta che la funzione dei media, inclusa la creazione di forum per il pubblico dibattito, non è limitata ai media o ai giornalisti professionisti. Infatti, caso in questione, la preparazione di un forum di dibattito pubblico era stata curata da una ONG. La Corte ha riconosciuto il contributo importante della società civile per la discussione di affari pubblici qualificando l'organizzazione ricorrente, la quale è coinvolta in controversie legate ai diritti umani, come "guardiano sociale". In dette circostanze, le attività del ricorrente legittimano una protezione da parte della CEDU analoga a quella accordata ai media. Inoltre, data l'intenzione del ricorrente di fornire le informazioni richieste al pubblico, contribuendo, di conseguenza, al dibattito pubblico sulla normativa che disciplina i reati legati agli stupefacenti, veniva chiaramente danneggiato il suo diritto di fornire informazioni. ${ }^{61}$

Nella sentenza Kenedi c. Ungheria la Corte ha ritenuto all'unanimità che vi fosse stata una violazione della CEDU per via dei procedimenti eccessivamente lunghi, durati oltre dieci anni, durante i quali il sig. Kenedi aveva tentato e ribadito il proprio accesso a documenti riguardanti i servizi segreti ungheresi. La Corte ha altresì ripetuto che

l'accesso a fonti documentarie originali per ragioni di legittima ricerca storica configurava un elemento essenziale dell'esercizio, da parte del ricorrente, del proprio diritto alla libertà di espressione.

La Corte ha fatto presente che Kenedi aveva ottenuto una sentenza della Corte che gli garantiva accesso ai documenti in questione, secondo la quale le corti ungheresi avevano ripetutamente deciso a suo favore i relativi processi. Le autorità ungheresi si erano persistentemente opposte al loro obbligo di conformarsi ai giudicati delle corti ungheresi, impedendo, di conseguenza, l'accesso ai documenti da parte di Kenedi, di cui egli aveva bisogno per redigere le proprie osservazioni. La Corte è giunta alla conclusione che le autorità ungheresi avevano agito in modo arbitrario e disobbedito

61 Társaság a Szabadságjogokért c. Ungheria. 
alla normativa nazionale. Ha inoltre stabilito che avevano abusato dei propri poteri ritardando l'esercizio, da parte di Kenedi, del proprio diritto alla libertà di espressione, in violazione dell'art. 10 della CEDU. ${ }^{62}$

Nella sentenza della Grande Camera Gillberg c. Svezia, la Corte ha riconosciuto che coloro che richiedevano le informazioni, in forma di dati scientifici, godevano del diritto di accesso a tali informazioni protetto dall'art. 10 della CEDU, il quale contribuirebbe "al libero scambio di opinioni e idee e ad una gestione efficiente e corretta dei pubblici affari". ${ }^{63}$

Più recentemente, la Corte europea ha ripetuto nel caso Youth Initiative for Human Rights c. Serbia che:

la ricerca di informazioni rappresenta una fase preparatoria essenziale nel giornalismo e un elemento intrinseco e protetto della libertà di stampa

e che:

gli ostacoli creati al fine di impedire l'accesso ad informazioni di pubblico interesse potrebbe scoraggiare coloro che lavorano nel settore mediatico o in campi ad esso affini dal perseguire un tale fine. Di conseguenza, potrebbero non essere più in grado di svolgere il loro ruolo vitale di "pubblici guardiani" e la loro capacità di fornire informazioni accurate e affidabili risulterebbe compromessa.

In riferimento alla sentenza TASZ c. Ungheria la Corte ha stabilito esplicitamente che "la nozione di 'libertà di ottenere informazioni' comprende il diritto di accedere alle informazioni”. La Corte è dell'opinione che, essendo la ONG ricorrente, la Youth Initiative for Human Rights, ovviamente coinvolta nella legittima ricerca di informazioni di pubblico interesse con l'intenzione di fornire tali informazioni al pubblico e di contribuire, di conseguenza, al pubblico dibattito, vi era stata un'interferenza con il suo diritto alla libertà di espressione. La ONG ricorrente richiese ai servizi segreti serbi di fornirle alcune informazioni relativi ai fatti legati all'uso di misure di sorveglianza elettronica. I servizi segreti avevano rigettato la ri-

62 Kenedi c. Ungheria. La Corte arrivò alla conclusione che, in questo caso, anche l'art. 13 (rimedio effettivo) era stato violato, in quanto il sistema ungherese non forniva un modo efficace di porre rimedio alle violazioni della libertà di espressione in questa situazione. La Corte ritenne inefficace la procedura disponibile a quel tempo in Ungheria per porre rimedio alle violazione dei diritti ex art. 10 subita da Kenedi. Vi era stata, di conseguenza, una violazione dell'art. 13 in combinazione con l'art. 10 della Convenzione.

63 Gillberg c. Svezia. 
chiesta facendo riferimento alle previsioni contenute nell'ordinamento serbo applicabili alle informazioni segreti. La Youth Initiative for Human Rights ha lamentato tale rifiuto di garantire l'accesso alle informazioni richieste detenute dai servizi segreti dinanzi alla Corte, malgrado un giudizio finale e vincolante da parte del Commissario per l'informazione a suo favore. La Corte ha ritenuto che le restrizioni imposte da parte dei servizi segreti serbi, ovvero il rifiuto di fornire accesso a documenti pubblici, non soddisfacessero il criterio di essere "previste dalla legge". La Corte ha ritenuto che "l'ostinata riluttanza da parte dei servizi segreti serbi di eseguire l'ordine del Commissario per l'informazione configurasse una violazione delle normative nazionali pari a un comportamento arbitrario, e che vi fosse stata, di conseguenza, una lesione dell'art. 10 della CEDU. La Corte ordinò alla Serbia di assicurare che fossero fornite al ricorrente le informazioni richieste da parte dei servizi segreti serbi entro tre mesi. ${ }^{64}$

Il riconoscimento, da parte della Corte, dell'applicabilità del diritto alla libertà di espressione e informazione in questioni di accesso ai documenti ufficiali rappresenta indubbiamente un importante nuovo sviluppo che espande il campo di applicazione dell'art. 10 della CEDU. ${ }^{65}$ Un tale approccio è, inoltre, pienamente in linea con il Commento Generale n. 34 del Comitato ONU per i diritti umani, il quale prevede che l'art. 19 del Patto internazionale sui diritti civili e politici "comprende il diritto di accesso alle informazioni detenute dalle pubbliche autorità". ${ }^{66}$

Nella casistica più recente, la Corte ha creato un livello di garanzia a parte per un diritto di accesso ai pubblici documenti, specialmente quando il ricorrente è coinvolto in una legittima ricerca di informazioni di pubblico interesse con l'obiettivo di contribuire al pubblico dibattito. ${ }^{67}$ La Corte ha manifestato un approccio simile nel caso Roşiianu c. Romania ribadendo che la raccolta di informazioni e la garanzia dell'accesso ai documenti detenuti dalle pubbliche autorità rappresentano un diritto cruciale per i gior-

\section{Youth Initiative for Human Rights c. Serbia.}

65 Vedi anche Tiilikka P. (2013) e la Convenzione sull'accesso a documenti ufficiali del Consiglio d'Europa, CETS n. 205. Disponibile a: www.conventions.coe.int $/ \mathrm{Tr}$ eaty/Commun/QueVoulezVous. asp?NT $=205 \& C M=8 \& D F=24 / 09 / 2012 \& C L=E N$ $\mathrm{G}$.

66 Comitato per i Diritti Umani delle Nazioni Unite (2011). Il Commento generale indica anche che "per conferire un effetto al diritto di accedere alle informazioni, gli Stati parte dovrebbero divulgare le informazione governative di interesse pubblico. Gli Stati dovrebbero adoperarsi per assicurare un facile, proprio, effettivo e pratico accesso a queste informazioni” (n. 19).

67 Österreichische Vereinigung zur Erhaltung, Stärkung und Schaffung eines wirtschaftlich gesunden land- und forstwirtschaftlichen Grundbesitzes c. Austria e Guseva c. Bulgaria. 
nalisti affinché essi siano in grado di riferire su questioni di pubblico interesse, ovvero aiutando ad implementare il diritto del pubblico affinché esso sia adeguatamente informato su tali questioni. La sentenza della Corte ha chiarito che sono necessari efficienti meccanismi di applicazione al fine di rendere praticabile ed effettivo il diritto di accedere ai pubblici documenti per scopi giornalistici in base all'art. 10 della CEDU. La Corte non accetterà restrizioni arbitrarie al diritto di accesso ai pubblici documenti, in quanto esse diverrebbero una forma di censura indiretta. Ricercare informazioni rappresenta un'operazione fondamentale nel giornalismo nonché un elemento intrinseco e protetto della libertà di stampa. Posto che l'intenzione del giornalista consiste nel comunicare le informazioni in questione al pubblico e, di conseguenza, nel contribuire al pubblico dibattito sulla buona governance pubblica, è stato chiaramente danneggiato il diritto di divulgare informazioni. La Corte ha altresì fatto presente che la complessità delle informazioni richieste ed il considerevole impegno per selezionare o compilare i documenti richiesti erano stati citati solamente per spiegare l'impossibilità di fornire tali informazioni in tempi rapidi, ma non possono essere portate in giudizio come argomento sufficiente o pertinente per rifiutare l'accesso ai documenti richiesti in toto.$^{68}$ Nella sentenza Guseva c. Bulgaria del 17 febbraio 2015, la Corte ha ritenuto che:

la ricerca di informazioni, tenendo in considerazione la loro susseguente divulgazione al pubblico, può ritenersi compresa all'interno della libertà di espressione del ricorrente garantita dall'art. 10 della CEDU ... non fornendo le informazioni richieste dal ricorrente, il sindaco ha interferito nella fase preparatoria del processo di informazione del pubblico creando un ostacolo amministrativo ... Il diritto del ricorrente di divulgare informazioni è stato, di conseguenza, leso. ${ }^{69}$

Nel presente caso, la Corte è giunta alla conclusione che la legge bulgara non prevedeva un arco temporale chiaro per attuare il diritto di accesso ai pubblici documenti e che la questione era lasciata alla buona volontà dell'autorità amministrativa responsabile dell'esecuzione della sentenza che ordinava la trasmissione dei documenti richiesti. La Corte ha ritenuto che una tale mancanza di un periodo certo per l'attuazione creasse incertezze in merito alle probabili tempistiche di attuazione la quale non si è, infine, mai concretizzata. Di conseguenza, la normativa nazionale applicabile di- 
fettava della capacità previsionale richiesta per superare la valutazione della Corte $e x$ art. 10(2) CEDU.

In ogni caso, alcune decisioni della Corte hanno creato dei dubbi circa l'ambito di applicazione e gli sviluppi futuri del diritto di accesso ai documenti detenuti dalle pubbliche autorità. In una decisione recente, pronunciata da un collegio formato da tre giudici, non è stata ravvisata alcuna violazione dell'art. 10 della CEDU in riferimento al rigetto della richiesta del ricorrente nei confronti di un'amministrazione comunale di fornire la lista dei pagamenti effettuati con i fondi comunali nei confronti di partiti politici, gruppi parlamentari e fondazioni politiche negli anni 2000, 2001 e 2002. Il ricorrente richiedeva anche informazioni sui pagamenti effettuati verso partito politici da parte di società detenute dal comune. ${ }^{70}$ La Corte ha fatto presente che il ricorrente stava ricercando informazioni di pubblico interesse ed ha assunto che egli volesse divulgarle al pubblico. La Corte non ha ritenuto necessario stabilire se il ricorrente andasse qualificato o meno come membro della stampa o se il proprio lavoro potesse essere considerato o meno simile a quello di una ONG quando si tratta di ricercare informazioni. La sentenza della Corte fa riferimento alla propria sentenza nel caso Östereichische Vereinigung zur Erhaltung, Stärkung und Schaffung c. Austria del 28 novembre 2013, nella quale ha:

assunto un diritto di accesso alle informazioni in un caso in cui le autorità non avevano pubblicato informazioni rilevanti di notevole interesse pubblico in un database elettronico o in altra forma.

In ogni caso, la Corte ha proseguito, facendo riferimento alla propria casistica (Leander c. Svezia 1987 e Guerra c. Italia 1998), affermando che:

Nel contesto specifico dell'accesso alle informazioni, la Corte ha ritenuto che il diritto di ottenere informazioni proibisce, in sostanza, ad un governo di impedire ad una persona di ottenere informazioni che altri vogliono o vorrebbero fornirle ... Ha altresì ritenuto che il diritto di ricevere informazioni non possa essere interpretato come obbligo positivo per lo Stato di raccogliere e divulgare egli stesso informazioni ... Di conseguenza, la Corte non ritiene che possa essere desunto dalla propria casistica un obbligo generale, nei confronti dello Stato, di fornire informazioni in forma specifica fondato sull'art. 10, in particolare quando, come nel caso in questione, ciò comporterebbe un carico di lavoro significativo.

70 Friedrich Weber c. Germania. 
La decisione mette a fuoco le differenze col caso TASZ c. Ungheria, poiché, in tale caso,

la Corte ha tenuto in considerazione il fatto che le informazioni richieste erano "pronte e disponibili" e non richiedevano la raccolta di dati di alcun genere da parte del governo.

Sulla base di questo ragionamento ambiguo la Corte, nella sentenza Friedrich Weber c. Germania, è giunta alla conclusione che

nel presente caso, indipendentemente dal proprio possibile status di membro della stampa, non vi è stata alcuna interferenza con il diritto del ricorrente di ottenere e di divulgare informazioni garantito dall'art. 10, co. 1 della Convenzione. ${ }^{71}$

In ogni caso, sulla base dei giudicati nei casi TASZ c. Ungheria, Kenedi $c$. Ungheria, Gillberg c. Svezia, Österreichische Vereinigung zur Erhaltung, Stärkung und Schaffung c. Austria, Youth Initiative for Human Rights c. Serbia, Roşiianu c. Romania e Guseva c. Bulgaria, si può concludere che l'art. 10 della CEDU tutela il diritto di accedere ai pubblici documenti quando:

(I) le informazioni sono detenute da una pubblica autorità in regime di monopolio informativo;

(II) le informazioni andrebbero rivelate nell'interesse pubblico; e

(III) il richiedente sta agendo in funzione di pubblico guardiano.

Come affermato dalla Corte, "la libertà di ricevere informazioni comprende il diritto di accedere alle informazioni". Tale diritto, come mostrato nella sentenza Youth Initiative for Human Rights c. Serbia, può anche includere il diritto di ottenere accesso ai documenti di servizi segreti e delle proprie attività di sorveglianza. La Corte può addirittura ordinare alle autorità di uno Stato membro di costringere i servizi segreti a fornire ad un giornalista $\mathrm{o}$ ad una ONG le informazioni richieste. ${ }^{72}$

\section{LA PROTEZIONE DEI WHISTLEBLOWER}

La Corte ha aggiunto un ulteriore elemento cruciale al fine di promuovere la trasparenza e di assicurare che i media possano svolgere il loro ruolo di pubblici guardiani in una società democratica, riportando i fatti di pubbli-

71 Vedi anche Shapovalov c. Ucraina.

72 Youth Initiative for Human Rights c. Serbia. 
co interesse. Dopo aver sviluppato un elevato livello di protezione per le fonti giornalistiche al fine di mantenere riservate le identità delle fonti giornalistiche, la Corte ha iniziato a proteggere i whistleblower direttamente sulla base dell'art. 10 della CEDU, tutelando il loro diritto alla libertà di espressione. I whistleblower sono essenzialmente coloro che rivelano cattive condotte, episodi di frode, corruzione, mala gestione $\mathrm{o}$ attività disoneste $\mathrm{o}$ illegali all'interno di un'azienda, un'amministrazione o un'organizzazione privata o pubblica. I whistleblower riportano violazioni dell'integrità morale e criticano, di conseguenza, molto spesso datori di lavoro, aziende o gruppi direzionali. In molti casi infrangono (anche) l'obbligo di confidenzialità o un obbligo di segretezza, specialmente quando le violazioni di integrità morale vengono riferite ai giornalisti o ai media.

La protezione dei whistleblower e il diritto dei giornalisti di proteggere le loro fonti

La protezione delle fonti giornalistiche è un modo indiretto per mettere al riparo i whistleblower da persecuzioni o rappresaglie per aver rivelato informazioni ai giornalisti o ai media in casi in cui è in gioco l'interesse pubblico, per esempio in casi di corruzione, frode $\mathrm{o}$ attività illecite.

Secondo la Corte:

La protezione delle fonti giornalistiche è una delle condizioni fondamentali per la libertà di stampa, come riconosciuto e ribadito in vari strumenti internazionali inclusa la Raccomandazione del Comitato dei Ministri ... Senza una tale protezione, le fonti potrebbero essere scoraggiate dal sostenere la stampa nell'informare il pubblico su questioni di pubblico interesse. Di conseguenza, verrebbe affievolito il ruolo vitale di pubblico guardiano della stampa e compromessa la capacità della stampa di fornire informazioni accurate e affidabili. Prestando attenzione all'importanza della protezione delle fonti giornalistiche per la libertà di stampa in una società democratica ed il potenziale "effetto dissuasivo" che un ordine di rivelazione di tali fonti ha sull'esercizio di tale libertà, una misura del genere non può ritenersi compatibile con l'articolo 10 della Convenzione, a meno che essa non sia giustificata da un'esigenza preponderante nel pubblico interesse. ${ }^{73}$

73 Goodwin c. Regno Unito. 
Le interferenze con il diritto di proteggere le fonti giornalistiche possono essere giustificate soltanto quando è garantito il rispetto di severe garanzie sostanziali e procedurali. La Corte può emettere un ordine di rivelazione delle fonti soltanto al fine di soddisfare una "esigenza preponderante nel pubblico interesse", per esempio per prevenire o investigare reati più gravi o violenza (razziale), proteggere il diritto alla vita o prevenire abusi sessuali sui minori classificabili come trattamento disumano o degradante. ${ }^{74}$

In varie occasioni, la Corte ha ritenuto che le perquisizioni delle case o dei luoghi di lavoro dei giornalisti configurino una violazione dell'art. 10 della CEDU e dei principi di sussidiarietà e di proporzionalità. ${ }^{75} \mathrm{La}$ casistica della Corte dimostra che, in vari casi, il diritto dei giornalisti di tutelare le loro fonti ha evitato che il datore di lavoro del whistleblower, sia nel settore pubblico quanto nel settore privato, venisse a conoscenza dell'identità del dipendente che aveva presumibilmente rivelato informazioni riservate di pubblico interesse ad un giornalista. Nelle sentenze Goodwin c. Regno Unito e Financial Times Ltd. e altri c. Regno Unito la Corte ha ritenuto che i tentativi di rivelare le identità delle fonti giornalistiche che avevano rivelato informazioni sull'azienda ledessero l'art. 10 della CEDU, così come lo furono le perquisizioni e le confische effettuate in redazioni e nelle abitazioni private dei giornalisti con lo scopo di identificare un "presumibile" funzionario o dipendente pubblico, per esempio nei casi Schmit c. Lussemburgo, Tillack c. Belgio e Nagla c. Lettonia.

74 Nordisk Film \& TV A/S c. Danimarca e Šečič c. Croazia. Vedia anche Stichting Ostade Blade c. Olanda.

In questa ultima decisione la Corte non rilevò violazioni dell'art. 10 del CEDU, considerando che la ricerca e confisca di computer e di altri materiali editoriali e dati fosse giustificata dal fatto che le autorità giudiziarie stessero provando ad identificare il perpetratore di una serie di attacchi bomba e che avessero buone ragioni di credere che il materiale confiscato nell'ufficio editoriale potesse essere d'aiuto per le indagini.

75 Roemen e Schmit c. Lussemburgo; Ernst ed altri c. Belgio; Voskuil c. Olanda; Tillack c. Belgio; Financial Times Ltd. ed altri c. Regno Unito; Sanoma Uitgevers BV c. Olanda; Martin ed altri c. Francia; Ressiot ed altri c. Francia; Telegraaf Media Nederland Landelijke Media N.V. ed altri c. Olanda; Saint-Paul Luxembourg S.A. c. Lussemburgo e Nagla c. Lettonia. Vedi anche Comitato dei Ministri del Consiglio d'Europa e Racommendazione n. R (2000) 7 sul diritto dei giornalisti alla non divulgazione delle fonti di informazione, 8 marzo 2000, www.coe.int/t/dghl/standardsetting/medi a/; Assemblea parlamentare del Consiglio d'Europa, Raccomandazione 1950 (2011) sulla protezione dell fonti giornalistiche 25 gennaio 2011, http://assembly.c oe.int/mainf.asp?Link=/documents/adoptedtext/ta11/erec1950.htm. 


\section{La protezione del whistle-blowing ed il diritto alla libertà di espressione}

Oltre e al di sopra di tale protezione indiretta dei whistleblower per mezzo del riconoscimento e dell'applicazione del diritto, per i giornalisti, di tutelare le loro fonti, la casistica recente della Corte ha aggiunto una protezione sostanziale diretta dei whistleblower. Infatti, mentre nella maggior parte dei Paesi europei non esiste una valida o effettiva protezione dei whistleblower, ${ }^{76}$ la Corte ha cercato di porre rimedio a questa situazione assicurando la tutela dei whistleblower in base all'art. 10 della CEDU. Nella sentenza Guya c. Moldavia, la Grande Camera ha ritenuto che il licenziamento di un funzionario pubblico che aveva rivelato informazioni, nello caso specifico tramite una lettera alla stampa, costituisse una restrizione illegittima del diritto alla libertà di espressione. ${ }^{77}$ In altri casi più recenti, la Corte ha ritenuto che vi fossero state lesioni dell'art. 10 della CEDU laddove i whistleblower avevano subito interferenze nel loro diritto alla libertà di espressione, inclusi i casi di rivelazione di informazioni confidenziali ai media.

\section{Il caso Guja: sei criteri per il whistle-blowing ${ }^{78}$}

In Moldavia, due politici, il Vicepresidente del Parlamento e il Viceministro degli Interni, avevano inviato una lettera al Procuratore generale chiedendogli di far cadere tutte le accuse in un'investigazione penale contro quattro poliziotti. Il sig. Guja, il capo dell'ufficio stampa del dipartimento del Procuratore generale, inviò una copia della lettera ad un giornale quale chiaro esempio di pressioni politiche esercitate sulla magistratura. La lettera costituì la base di un articolo in cui i due politici vennero accusati di interferire in un'indagine penale in corso. Fu presto chiaro che era stato Guja a inviare la lettera al giornale e furono avviati procedimenti disciplinari contro di lui. Guja disse al Procuratore generale di aver inviato la lettera in quanto convinto di poter aiutare ad evitare tale illegittima procedura. Nonostante le proprie nobili intenzioni venne licenziato.

76 Stevenson e Levi (2012). Questo studio raccomanda che lo Stato dovrebbe "attuare, o migliorare, le leggi nazionali sulla protezione dei lavoratori nei confronti di rappresaglie in circostanze dove i lavoratori stessi promuovano la divulgazione di informazioni reperite durante il lavoro, la cui divulgazione stessa è di pubblico interesse".

77 Guja c. Moldavia.

78 Per un commento vedi Junod (2009) e Voorhoof e Gombeer (2008). 
Il caso in questione presenta una situazione molto peculiare: l'esercizio della libertà di espressione in un caso di corruzione. Nella propria sentenza, la Corte ha citato i trattati ONU ratificati dalla Moldavia e i Trattati del Consiglio d'Europa che tutelano le persone (inclusi gli impiegati) che denunciano la corruzione. Ha altresì citato la Convenzione OIL n. 158, il cui art. 5 afferma che:

il fatto di aver presentato un'istanza o partecipato a procedure avviate contro un datore di lavoro in ragione di presunte violazioni della legislazione, o presentato un ricorso alle autorità amministrative competenti

non costituisce motivo valido di licenziamento.

Tenendo conto del fatto che Guja era un funzionario pubblico, i principi sostenuti dalla Corte in altre sentenze relative al diritto alla libertà di espressione dei funzionari pubblici erano, mutatis mutandis, applicabili al caso in questione. In ogni caso, la Corte ha compiuto una leggera differenziazione trattandosi di un caso di whistle-blowing. La Corte ha affermato soprattutto che:

un funzionario pubblico può, durante il proprio lavoro, giungere a conoscenza di informazioni interne, incluse informazioni segrete, la cui divulgazione o pubblicazione corrisponde ad un forte interesse pubblico. ${ }^{79}$

La Corte ha pertanto riconosciuto che, in determinate circostanze, la denuncia di illeciti debba essere protetta, per esempio quando un funzionario pubblico è l'unica persona o una delle poche persone a conoscenza di ciò che sta accadendo sul posto di lavoro e se lui/lei si trova nella posizione migliore per rivelarlo. ${ }^{80}$ In ogni caso, posto che i funzionari pubblici sono tenuti alla discrezionalità o confidenzialità, l'impiegato dovrebbe prima informare il proprio superiore/i propri superiori. Rendere pubblica l'informazione o rivelarla ai media è consentito soltanto come ultima istanza $(u l$ timum remedium) ${ }^{81}$ Di conseguenza, nel caso Guja c. Moldavia, era necessario esaminare se fosse stato possibile o meno comunicare le informazioni in altro modo al fine di rivelare e porre rimedio all'illecito in questione. La Corte ha imposto la condizione che un dovere interno di riferire debba altresì costituire un meccanismo effettivo per porre rimedio all'illecito:

79 Guja c. Moldavia.

80 Vedi inoltre Marchenko c. Ucraina, $\mathbb{} 46$.

81 Guja c. Moldavia, $\mathbb{} 73$. 
Nel valutare se la restrizione della libertà di espressione sia avvenuta in maniera proporzionata la Corte deve, di conseguenza, prendere in considerazione se il ricorrente disponesse di un qualsiasi altro mezzo effettivo per porre rimedio all'illecito che intendeva rivelare.

Oltre a questa condizione di mancanza di alternativa efficace (1), vi sono alcuni altri fattori da tenere in considerazione: ${ }^{82}(2)$ deve essere in gioco un interesse pubblico; (3) l'informazione rivelata deve essere vera e precisa; (4) il danno producibile dall'informazione e il pubblico interesse devono essere bilanciati; (5) alla base dei motivi per la rivelazione deve trovarsi la buona fede; e (6) la sanzione imposta deve essere proporzionata.

Tenendo in considerazione tutti questi criteri, la Corte ha concluso che il licenziamento di Guja configurò una violazione del proprio diritto alla libertà di espressione e specialmente del proprio diritto di divulgare informazioni. ${ }^{83}$

La Corte ha formulato le proprie conclusioni nel modo seguente:

Essendo consapevole dell'importanza del diritto alla libertà di espressione in questioni di interesse generali, del diritto dei funzionari pubblici e di altri impiegati di rivelare comportamenti illegali e illeciti sul loro posto di lavoro, dei doveri e delle responsabilità degli impiegati nei confronti dei loro datori di lavoro e del diritto dei datori di lavoro di gestire il proprio personale e avendo bilanciato gli altri vari interessi coinvolti nel presente caso, la Corte giunge alla conclusione che l'interferenza nel diritto alla libertà di espressione del ricorrente, in particolare del suo diritto di fornire informazione, non era "necessaria in una società democratica". Di conseguenza, vi fu una violazione dell'art. 10 della Convenzione.

Altri casi di whistleblower in cui la Corte ha rilevato una violazione dell'art. 10 della CEDU

A partire dalla sentenza Guja, il whistle-blowing da parte di funzionari pubblici, funzionari governativi e addirittura da parte di magistrati e dipendenti dei servizi segreti militari viene effettivamente tutelato in forza dell'art. 10 della CEDU. La sentenza Kayasu c. Turchia riguardava la sanzione disciplinare e la condanna penale di un pubblico ministero il quale aveva

82 Ibid., $\$ \$ \$ 74-78$.

83 Ibid., $\$ 97$. 
presentato, come cittadino, un'istanza all'Ufficio del Pubblico ministero della Corte di sicurezza statale in cui accusava due militari di alto rango in congedo di essere coinvolti in un golpe militare. Il pubblico ministero aveva anche trasmesso il testo dell'istanza ai media, che lo avevano successivamente diffuso. Le autorità turche ritenettero il testo della petizione lesivo dei doveri professionali del pubblico ministero, che esso screditasse le istituzioni dello Stato in modo oltraggioso e che danneggiasse la reputazione degli alti ufficiali militari. Ad ogni modo, la Corte ha evidenziato che "il dibattito in questione è servito in sostanza a dimostrare un malfunzionamento del regime democratico". Data la gravità delle sanzioni la Corte ha concluso che l'interferenza nel diritto alla libertà di espressione del pubblico ministero configurasse una violazione dell'art. 10 della CEDU. ${ }^{84}$

Anche il caso Kudeshkina c. Russia riguardava una forma di whistle-blowing. ${ }^{85} \mathrm{Nel} 2005$, Olga Borisovna Kudeshikina si è rivolta alla Corte in seguito al suo licenziamento dal ruolo di giudice della corte cittadina di Mosca, presso la quale aveva svolto servizio per oltre 18 anni. Venne rimossa dal proprio incarico da un tribunale disciplinare a causa di una serie di espressioni rese ai media in cui affermava che le era stato levato un caso in cui stava indagando su fenomeni di corruzione e frode finanziaria di larga scala. Kudeshikina rese tali affermazioni dopo essere stata sospesa dal proprio ruolo di giudice, su sua richiesta, perché si era candidata alle elezioni del parlamento. In varie interviste legate alla campagna elettorale aveva riferito di manipolazioni ed interventi da parte di funzionari di alto rango, uomini d'affari e politici che esercitavano sistematicamente pressioni sulla corte moscovita. Nella propria campagna elettorale si è espressa a favore di un'accurata riforma della giustizia tenendo conto di una performance migliore e di un potere giudiziario più indipendente. In ogni modo, non venne eletta alla Duma e poco dopo aver ripreso il proprio ruolo di giudice venne licenziata. La Corte ha ritenuto che il licenziamento di Kudeshkina, dovuto alle sue esternazioni pubbliche, configurasse una violazione dell'art. 10 della CEDU, il quale accorda a tutti, inclusi i funzionari pubblici ed i magistrati, il diritto alla libertà di espressione. La sentenza ha chiarito che la Corte non ha considerato la presunta violazione della riservatezza professionale e la divulgazione di informazioni false una giustificazione convincente per il suo allontanamento. Kudeshkina non aveva infatti pubblicato informazioni concrete su processi penali in corso e le sue asserzioni non potevano essere ritenute attacchi personali ed infondati nei confronti

84 Kayasu c. Turchia.

85 Kudeshkina c. Russia, $\$ 99$. 
di singoli giudici o del potere giudiziario nel suo complesso, ma piuttosto esternazioni rilevanti ed imparziali su una questione di interesse pubblico.

La Corte ha evidenziato che:

La ricorrente ha reso la critica pubblica in riferimento a una materia altamente sensibile, ovvero il comportamento di vari funzionari che affrontavano un caso di corruzione su ampia scala in cui essa ricopriva il ruolo di giudice. Infatti, le sue interviste si riferivano a uno stato delle cose sconvolgente e asserivano che casi di pressioni sui giudici erano comuni e che questo problema andava trattato seriamente se si volesse conservare l'indipendenza del potere giudiziario e affinché questo continuasse a godere della stima pubblica. Non vi è alcun dubbio sul fatto che, così facendo, abbia sollevato una tematica di pubblico interesse molto importante, la quale dovrebbe essere aperta a un libero dibattito in una società democratica. La sua decisione di rendere pubbliche tali informazioni era basata sulla sua esperienza personale e venne presa solo dopo che le era stato impedito di partecipare al processo in veste ufficiale. ${ }^{86}$

Nonostante si possano formulare alcuni dubbi circa la ferocità con la quale Kudeshkina ha formulato i propri punti di vista, la Corte ha ritenuto che le sue critiche ben fondate abbiano contribuito a un importante dibattito nella società.

Ad ogni modo, nonostante la ricorrente si fosse concessa un certo grado di esagerazione e generalizzazione, tipico della fase preelettorale, le sue esternazioni non erano completamente prive di fondamenti fattuali ... e, di conseguenza, non andavano ritenute attacchi gratuiti di natura personali bensì giusti commenti su un tema di grande importanza pubblica. ${ }^{87}$

Inoltre, la Corte ha ritenuto l'allontanamento di un giudice con un'esperienza di 18 anni una sanzione sproporzionata, a maggior ragione in quanto porterebbe altri magistrati ad astenersi dall'esprimere commenti critici sul funzionamento del potere giudiziario e della politica giudiziaria in futuro. Di nuovo, la Corte ha posto l'accento sull'"effetto dissuasivo", il cui risultato consiste nel fatto che un soggetto non oserebbe più rendere un'esternazione pubblica per paura di sanzioni. Ha sottolineato che un tale "effetto dissuasivo" è deleterio per una democrazia e che Kudeshkina godeva

86 Kudeshkina c. Russia, $\$ 94$.

87 Kudeshkina c. Russia, $\$ 95$. 
certamente del diritto di sottoporre all'attenzione pubblica le tematiche denunciate.

Il messaggio della Corte è chiaro: i magistrati (russi) i quali contribuiscono al pubblico dibattito nei media sulla manipolazione del potere giudiziario andrebbero sostenuti anziché sanzionati con l'allontanamento. Sfortunatamente, le autorità russe si sono rifiutate di riaprire i procedimenti riguardanti l'allontanamento di Kudeshkina dalla magistratura. Di conseguenza, nonostante la Corte avesse ritenuto che le autorità russe avevano leso il diritto alla libertà di espressione della ricorrente garantito dall'art. 10 della CEDU, tale caso mostra la difficoltà di applicare efficacemente le decisioni della Corte sulla protezione dei whistleblower in alcuni Stati membri della CEDU. 88

Altre sentenze mostrano che lo scopo della casistica della Corte consiste nello stimolare la rivelazione o la copertura di (gravi) illeciti o violazioni, in particolare in situazioni in cui solo una o poche persone ed impiegati ne sono informate. ${ }^{89}$ Nella sentenza Marchenko c. Ucraina la Corte ha evidenziato, similmente alla sentenza Guja c. Moldavia, che:

la segnalazione di condotte illecite o violazioni nel settore pubblico deve essere protetta, in particolare in quanto solo un gruppo di persone ristretto era a conoscenza di quanto accaduto. ${ }^{90}$

Anche nel caso Frankovicz c. Polonia la Corte ha rilevato una violazione dell'art. 10 della CEDU, questa volta sulla base di una sanzione disciplinare inflitta ad un dottore il quale aveva espresso commenti negativi in un report medico su un paziente relativi al trattamento e la cura dello stesso in un determinato ospedale. ${ }^{91}$ La Corte ha aggiunto che il report non contene-

88 Olga Borisovna Kudeshkina c. Russia (n. 2), e Kudeshkina c. Russia. Ai sensi dell'art.46 $\$ 2$ della CEDU, il Comitato dei Ministri è investito del potere di supervisionare l'esecuzione dei giudizi della Corte e di valutare le misure adottate dagli Stati coinvolti. Alla luce dei sopracitati principi di diritto internazionale e delle informazioni fornite dallo Stato in questione, è compito del Comitato dei Ministri decidere se quest'ultimo ha ottemperato in buona fede ai propri doveri di ristabilire, nella misura possibile, la situazione precedente alla violazione. Mentre lo Stato in parola rimane in principio libero di scegliere i mezzi con cui adempire ai suoi doveri, rimane compito del Comitato dei Ministri dichiarare se questi mezzi scelti sono compatibili con le conclusioni stabilite dal giudizio della Corte il 26 febbraio 2009, al paragrafo 95. Riferendosi in particolare alla riapertura dei procedimenti, la Corte non ha competenza per ordinare tali misure.

89 Juppala c. Finlandia e Marchenko c. Ucraina.

90 Marchenko c. Ucraina, $\mathbb{} 46$.

91 Frankovicz c. Polonia, $\mathbb{\$} 51$. 
va un attacco personale gratuito ai colleghi, ma era fondato su dati sanitari riguardanti il trattamento medico di un paziente da parte di un altro dottore, facendo quindi notare che il report era legato ad un interesse pubblico. In queste circostanze, non era necessaria, in una società democratica, la sanzione disciplinare in forma di rimprovero. Venne infatti considerata, da parte della Corte, una violazione del diritto alla libertà di espressione del medico. Nella sentenza Sosinowska c. Polonia la Corte ha osservato, con un ragionamento simile, che il rimprovero di un dottore da parte di una corte medica corrispondesse ad una violazione del suo diritto alla libertà di espressione. La Corte ha ritenuto che le autorità polacche non erano state in grado di riconoscere che la dott.ssa Sosinowska aveva difeso un interesse socialmente giustificato avendo formulato una valutazione critica dal punto di vista medico riguardante temi di pubblico interesse. ${ }^{92}$

Nel caso Bucur e Thoma c. Romania la Corte ha ritenuto che l'interesse generale alla diffusione di informazioni ai media che hanno rilevato attività illecite all'interno dei servizi segreti rumeni fosse talmente importante in una società democratica da prevalere sull'interesse di mantenere il pubblico riserbo in tale istituzione. Applicando i sei "criteri Guja", la Corte non era convinta che un reclamo formale ad una commissione parlamentare sarebbe stato un mezzo efficace per contrastare le irregolarità all'interno dei servizi. Ha altresì osservato che le informazioni sulla sorveglianza illecita di giornalisti, politici e uomini di affari rivelata alla stampa colpiva le fondamenta democratiche dello stato rumeno. Di conseguenza, le informazioni riguardavano temi molto importanti per il dibattito politico in una società democratica, nei confronti dei quali l'opinione pubblica godeva di un interesse legittimo.

Il fatto che i dati e le informazioni in questioni fossero classificate come "ultra-top-secret" non costituiva una ragione sufficiente per interferire, nel presente caso, nel diritto del whistler-blower, e le misure adottate contro Bucur rischiavano oltretutto di sortire un "effetto dissuasivo". La condanna di Bucur a causa della rivelazione ai media delle informazioni circa le attività illegittime dei servizi segreti venne ritenuta lesiva dell'art. 10 della CEDU. ${ }^{93}$ Nella propria sentenza la Corte ha anche citato la Risoluzione

92 Sosinowska c. Polonia.

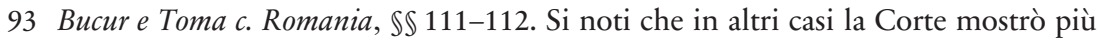
riguardo per informazioni segrete, classificate come informazioni militari: Pasko c. Russia, \$S 86-87. In Pasko c. Russia la Corte non ha applicato i criteri di Guja, le informazioni in questione riguardano seri problemi ambientali relativi all'inquinamento nucleare. 
1729(2010) dell'Assemblea parlamentare del Consiglio d'Europa in merito alla protezione dei whistleblower.

Anche i whistleblower nel settore privato possono invocare il loro diritto alla libertà di espressione se rivelano presunti comportamenti illeciti dei loro datori di lavoro. Anche nel caso Heinisch c. Germania la Corte ha applicato, mutatis mutandis, i criteri Guja. ${ }^{94}$

Mentre un tale obbligo di fedeltà può essere maggiore nel caso dei funzionari pubblici e degli impiegati del settore pubblico rispetto ai dipendenti nei rapporti di lavoro del settore privato, la Corte ritiene che esso costituisca senza dubbi un elemento di quest'ultima categoria di impiego. Condivide, di conseguenza, l'opinione del governo secondo i cui i principi ed i criteri stabiliti dalla casistica della Corte in riferimento al bilanciamento del diritto alla libertà di espressione del dipendente nel segnalare comportamenti illeciti o violazioni da parte del proprio datore di lavoro ed il diritto, di quest'ultimo, di proteggere la propria reputazione ed i propri interessi commerciali vadano applicati anche al caso in questione. La natura e l'estensione della fedeltà dovute dal dipendente in un particolare caso impattano sul bilanciamento dei diritti dell'impiegato e l'interesse confliggente del datore di lavoro. ${ }^{95}$

Altre sentenze della Corte riflettono chiaramente l'elevato livello di protezione del diritto alla libertà di espressione degli individui che partecipano al pubblico dibattito facendo affidamento a informazioni provenienti dal loro ambiente di lavoro, per esempio nei casi Wojtas-Kaleta c. Polonia ${ }^{96} \mathrm{e}$ Rubins c. Lettonia..$^{97}$ Questo approccio venne condiviso anche dalla Grande Camera della Corte nel caso Morice c. Francia,${ }^{98}$ in cui si ritenne che il ricorrente, Morice, un avvocato che aveva redatto un articolo pubblicato sul

$94 \mathrm{Al}$ tempo della sentenza Guja non era del tutto certo se quei principi dovessero essere applicati anche nei confronti dei dipendenti del settore privato, vedi Junod (2009:240).

95 Heinisch c. Germania, $\mathbb{} 64$.

96 Wojtas-Kaleta c. Polonia.

97 Rubins c. Lettonia. Questa sentenza è un altro esempio di intereferenza sproporzionata nel diritto di libertà d'espressione di un impiegato, in questo caso di un professore unversitario che esprimeva forti dubbi sulla politica aziendale e di gestione del datore di lavoro. La Corte dichiarò che il licenziamento dell'impiegato in questo caso "fosse imputabile di avere un serio effetto sugli altri impiegati dell'università e di scoraggarli da innalzare critiche" e che sanzioni così severe, con tali conseguenza, in luce del caso come un insieme, fossero difficili da giustificare in una società democratica.

98 Morice c. Francia. 
quotidiano Le Monde, avesse espresso giudizi di valore con un fondamento fattuale sufficiente e che le proprie osservazioni riguardanti un tema di pubblico interesse non avessero superato i limiti della sua libertà di espressione:

un avvocato dovrebbe essere in grado di porre l'attenzione pubblica su potenziali difetti del sistema giudiziario; il potere giudiziario può beneficiare di una critica costruttiva del genere.

La Grande Camera ha anche ritenuto che il rispetto per l'autorità del potere giudiziario non possa giustificare una restrizione illimitata del diritto alla libertà di espressione. Nonostante la difesa di un cliente da parte di un avvocato non vada svolta sui media, bensì all'interno delle aule di giustizia, utilizzando tutti i rimedi esperibili, la Grande Camera accetta che vi potrebbero essere "circostanze molto specifiche che giustificano il fatto che un avvocato renda un'affermazione pubblica sui media, come nel caso in questione".

Nella sentenza Matúz c. Ungheria la Corte ha nuovamente sottolineato con fermezza l'importanza della protezione dei whistleblower, nel caso specifico per un giornalista che aveva scosso l'opinione pubblica con il proprio libro nel quale asseriva l'esistenza di forme di censura all'interno dell'emittente pubblica ungherese. La Corte ha altresì confermato la gravità dell'allontanamento di un whistleblower per le proprie legittime attività svolte nel pubblico interesse. ${ }^{99}$ Di nuovo, la Corte ha fatto riferimento, e ha applicato, i sei criteri Guja (vedi sopra). Ha ribadito che il contenuto del libro di Matúz era essenzialmente di pubblico interesse ed ha confermato che non contestava che i documenti pubblicati da Matúz fossero autentici e che le sue osservazioni si basassero sui fatti. Proseguì statuendo che, tenendo in considerazione il ruolo svolto dai giornalisti nella società e le loro responsabilità nel contribuire ed incoraggiare il pubblico dibattito, l'obbligo di riservatezza e confidenzialità non può essere applicato ai giornalisti nel medesimo modo applicato nei confronti dei membri del pubblico, posto che la diffusione di informazioni e idee è intrinseca nella natura del giornalismo. La Corte ha altresì affermato che il giornalista aveva fatto riferimento a documenti riservati con la mera intenzione di avvalorare le proprie tesi sulla censura e che non vi era alcuna parvenza di attacchi personali gratuiti. Inoltre, la decisione di rendere pubblici le informazioni e i documenti era basata sull'esperienza: né le rimostranze rivolte al presidente del- 
l'emittente né le lettere inviate al consiglio di amministrazione hanno prodotto alcuna risposta. Di conseguenza, la Corte ha ritenuto che:

è sufficiente che la pubblicazione del libro sia avvenuta solo dopo che al ricorrente era stato impedito di porre rimedio alla percepita interferenza nel proprio lavoro giornalistico all'interno dell'emittente stessa; ciò è avvenuto per mancanza di canali di diffusione alternativi effettivi.

Infine, la Corte ha osservato che le corti ungheresi avevano ritenuto che il mero fatto che Matúz avesse pubblicato il libro fosse sufficiente per concludere che egli avesse agito per danneggiare il proprio datore di lavoro e che avesse, di conseguenza, violato il proprio contratto lavorativo. Le corti ungheresi avevano ignorato l'argomentazione di Matúz, secondo cui egli aveva esercitato la propria libertà di espressione nell'interesse pubblico. Inoltre, la sentenza della Corte suprema ungherese stabiliva esplicitamente che la vertenza era limitata ad un contenzioso lavorativo e non ad una presunta violazione dei diritti umani del ricorrente. Un tale approccio mostra che è stato ignorato il diritto umano alla libertà di espressione da parte delle corti ungheresi, le quali non hanno neppure esaminato in che modo la materia oggetto del libro di Matúz ed il contenuto della sua pubblicazione avrebbero comportato una restrizione della sua libertà di espressione. La Corte ha altresì ravvisato che "al ricorrente era stata imposta una sanzione alquanto severa", ovvero la risoluzione, con effetto immediato, del rapporto di lavoro, e concluso che l'interferenza con il diritto alla libertà di espressione del ricorrente non era necessaria in una società democratica:

Consapevole dell'importanza del diritto alla libertà di espressione su questioni di interesse generale, degli obblighi e delle responsabilità professionali del ricorrente in quanto giornalista da un lato e dei doveri e delle responsabilità dei dipendenti nei confronti del loro datore di lavoro dall'altro lato, e avendo bilanciato i vari interessi coinvolti nel caso, la Corte conclude che l'interferenza nel diritto alla libertà di espressione del ricorrente non era "necessaria in una società democratica”.

Di conseguenza, la Corte ha ritenuto all'unanimità che vi era stata una lesione dell'art. 10 della CEDU. ${ }^{100}$ La sentenza ha indubbiamente contribuito ad una maggiore consapevolezza in futuro relativa alla mancanza di protezione dei whistleblower in molti Paesi d'Europa. Allo stesso tempo, la casi-

100 Vedi inoltre: Voorhoof (2014c). Vedi inoltre: Comitato dei Ministri del Consiglio d'Europa (2014), Raccomandazione Rec(2002)2. 
stica della Corte ha elaborato un quadro per la protezione dei whistleblower basato sul diritto alla libertà di espressione, formulando criteri chiari:

1. Non è stato possibile per l'impiegato o il funzionario pubblico chiamare il proprio datore di lavoro, capo dipartimento o qualsiasi altra autorità per rivelare le illegittimità e porre loro rimedio?

2. Le informazioni si riferiscono ad illeciti gravi o ad una questione di rilevanza sociale?

3. Le informazioni rivelate erano autentiche, affidabili e precise?

4. Quale danno è stato inferto al datore di lavoro rivelando e divulgando documenti interni e riservati?

5. Quali sono state le motivazioni del whistleblower?

6. A quale tipo di sanzioni era esposto il whistleblower e quali sono le conseguenze delle stesse?

\section{Il whistle-blowing e la politica del Consiglio d'Europa}

L'Assemblea parlamentare del Consiglio d'Europa ha evidenziato, in linea con la casistica della Corte in merito all'applicazione dell'art. 10 della CEDU in casi di whistle-blowing, l'importanza di ciò nella propria Risoluzione $1729 / 2010$.

L'Assemblea parlamentare riconosce l'importanza dei whistleblower individui preoccupati che danno l'allarme per porre fine agli illeciti che mettono in rischio esseri umani vicini - in quanto le loro azioni offrono l'opportunità di rafforzare le responsabilità e sostengono la lotta alla corruzione ed alla mala gestione, sia nel settore pubblico che nel settore privato. I potenziali whistleblower vengono spesso scoraggiati per paura di ritorsioni o dalla mancanza di seguito alla loro denunce a danno del pubblico interesse ad una gestione efficiente e alla responsabilità degli affari pubblici e dell'economia privata. ${ }^{101}$

La risoluzione pone l'accento sui meccanismi di protezione per i whistleblower in conformità con una serie di principi base, come formulati nella casistica della Corte. La risoluzione punta a una "legislazione comprensiva" con un ampio campo di applicazione per proteggere i whistleblower, ${ }^{102}$ sia

101 Assemblea parlamentare del Consiglio d'Europa (2010a).

102 Assemblea parlamentare del consigio europeo (2010a): "6.1.1. la definizione di divulgazione protetta dovrebbe includere tutti gli accorgimenti sulla buona fede 
per i funzionari pubblici che per i dipendenti del settore privato. ${ }^{103} \mathrm{Si}$ insiste sulla necessità di un forte ancoraggio giuridico per i whistleblower, inter alia, nelle leggi sul lavoro, al fine di prevenire licenziamenti illegittimi o altre forme di rappresaglie relative all'impiego. Infine, gli Stati membri sono invitati a garantire la protezione dei whistleblower e a sviluppare meccanismi per proteggerli in maniera (più) adeguata. ${ }^{104}$

In una presa di posizione del 7 dicembre 2011, il Comitato dei Ministri del Consiglio d'Europa ha richiesto una migliore tutela giuridica per i whistleblower, inclusi coloro che utilizzano i media online e le nuove piattaforme digitali. Il Comitato dei Ministri ha evidenziato che:

Persone, ovvero rappresentanti della società civile, whistleblower e difensori dei diritti dell'uomo, fanno sempre maggiore affidamento ai social network, ai blog e altri mezzi di comunicazione di massa nel complesso per accedere a e scambiare informazioni, pubblicare contenuti, interagire, comunicare e socializzare tra di loro. Queste piattaforme stanno diventando parte integrale del nuovo ecosistema mediatico. Nonostante siano operate da privati, costituiscono una parte importante nella sfera pubblica in quanto facilitano il dibattito su tematiche di pubblico interesse. In alcuni casi possono svolgere, in modo simile ai media tradizionali, il ruolo di guardiani sociali ed hanno dimostrato la loro utilità nell'introdurre cambiamenti in positivo nella vita reale. ${ }^{105}$

Di conseguenza, il Comitato dei Ministri ha richiesto di adottare azioni in considerazione di un'effettiva protezione dei whistleblower in forza degli articoli 10 e 11 della CEDU. Nel frattempo, la giurisprudenza della Corte ha contribuito, applicando l'art. 10 della CEDU per proteggere i whistleblower, in maniera notevole alla tutela concreta degli individui i quali rivelano o divulgano, nel loro ambito lavorativo, informazioni su minacce all'interesse pubblico e che, di conseguenza, contribuiscono a rafforzare la trasparenza e la responsabilità democratica. In molte occasioni recenti (p.es. la co-

contro i vari tipi di azioni illecite, comprendendo tutte le principali violazioni dei diritti umani che minacciano o attentano alla vita, alla salute, alla libertà o a qualsiasi altro leggittimo interesse degli individui in quanto soggetti della pubblica amministrazione, o contribuenti, o azionisti, dipendenti o clienti di società private."

103 Assemblea parlamentare del Consiglio d'Europa (2010a): “6.1.2. la legislazione dovrebbe perciò tutelare i whistleblower sia del settore pubblico che di quello privato, includendo i membri di forze militari o dei servizi segreti."

104 Assemblea parlamentare del Consiglio d'Europa (2010b).

105 Comitato dei Ministri del Consiglio d'Europa (2011). 
pertura mediatica di "Lux Leaks" e "Swiss Leaks") è stata dimostrata l'importanza cruciale dei whistleblower per informare i media su importanti questioni di pubblico interesse.

Tale è altresì il messaggio della Raccomandazione del Comitato dei Ministri CM/Rec(2014)7 sulla protezione dei whistleblower del 30 aprile 2014 (la Raccomandazione), la quale riafferma che "la libertà di espressione ed i diritti di cercare e ottenere informazioni sono fondamentali per il funzionamento di una sana democrazia". La raccomandazione riconosce anche che:

gli individui che rivelano o divulgano informazioni su minacce o danni per il pubblico interesse ("whistleblower") possono contribuire a rafforzare la trasparenza e la responsabilità democratica

e fa esplicito riferimento al diritto alla libertà di espressione e di informazione garantite dall'art. 10 della CEDU. Prosegue raccomandando agli Stati membri di mettere in campo:

un quadro normativo, istituzionale e giurisdizionale per proteggere gli individui che rivelano o divulgano informazioni nel contesto delle loro relazioni lavorative su minacce o danni per l'interesse pubblico. ${ }^{106}$

$\mathrm{Al}$ fine di raggiungere tale scopo, le cornici giuridiche nazionali degli Stati membri dovrebbero favorire la creazione di un ambiente che incoraggi la rivelazione o la divulgazione in maniera aperto e gli individui dovrebbero sentirsi al sicuro nel trattare liberamente questioni di interesse pubblico. Si raccomanda di "mettere in campo canali chiari per coperture e rivelazioni nel pubblico interesse e il ricorso ad esse dovrebbe essere agevolato tramite misure adeguate". Tali canali per coperture e rilevazione comprendono:

- segnalazioni all'interno di un'organizzazione o azienda (incluse le persone destinate a ricevere le segnalazioni in forma confidenziale)

- segnalazioni ad agenzie di regolamentazione pubbliche significative, organi esescutivi e organi di controllo;

- divulgazioni al pubblico, per esempio ad un giornalista o ad un membro del Parlamento.

È importante che i whistleblower siano anche in grado di invocare una protezione giuridica e di fare affidamento al loro diritto alla libertà di espressione in base all'art. 10 della CEDU nei casi in cui forniscono informazioni

106 Comitato dei Ministri del Consiglio d'Europa (2014). 
al pubblico rilevando informazioni confidenziali ai media o ai giornalisti. ${ }^{107}$

È ovvio che la casistica della Corte non ha solo creato una tutela importante per il whistle-blowing basata sulla libertà di espressione, ha anche contribuito a rafforzare la consapevolezza circa la mancanza di protezione per i whistleblower in molti Paesi d'Europa. La raccomandazione richiede agli Stati membri di agire per stimolare, facilitare e proteggere il whistle-blowing e di mirare ad implementare a livello nazionale una soglia di protezione più elevata del whistle-blowing, svolto nel pubblico interesse, in linea con la casistica della Corte. ${ }^{108}$ L'Assemblea parlamentare del Consiglio d'Europa ha ribadito, in una Risoluzione del 23 giugno 2015 (la Risoluzione), l'importanza della casistica della Corte nel sostenere la libertà di parola e la protezione dei whistleblower. La risoluzione chiede agli Stati membri di:

convenire su uno strumento giuridico vincolante (convenzione) sulla protezione dei whistleblower sulla base della Raccomandazione del Comitato dei Ministri CM/Rec(2014)7, tenendo conto degli sviluppi recenti. ${ }^{109}$

La risoluzione pone anche l'accento sulla necessità di garantire la protezione dei whistleblower per i dipendenti della sicurezza nazionale o dei servizi segreti e di aziende private che lavorano in questo campo, in riferimento al pubblico interesse coinvolto nella sorveglianza di massa da parte dei servizi segreti e di sicurezza. Come stabilito dalla Corte nel caso Bucur e Thoma c. Romania, l'interesse generale alla divulgazione di informazioni che rivelavano attività illecite all'interno dei servizi segreti ai media fu così importante in una società democratica che prevalse sull'interesse nel mantenere il pubblico riserbo in tale istituzioni: le informazioni sulla sorveglianza illecita delle telecomunicazioni di giornalisti, politici e uomini d'affari rivelata ai media colpiva le fondamenta democratiche dello Stato. ${ }^{110}$

107 Si noti che attualmente questa tutela non è garantita dalle linee giuda dell'Unione europea sul whistleblowing, vedi: Bang (2015).

108 Comitato dei Ministri del Consiglio d'Europa (2014).

109 Assemblea parlamentare del Consiglio d'Europa (2015).

110 Bucur e Toma c. Romania, \$S 111-112. 


\section{BIBLIOGRAFIA}

Assemblea parlamentare del Consiglio d'Europa (2010a), Risoluzione 1729 (2010) relativa alla protezione dei "whistleblower", http://assembly.coe.int/nw/xml/XRef/ Xref-XML2HTMLen.asp?fileid=17851\&lang=en.

Assemblea parlamentare del Consiglio d'Europa (2010b), Raccomandazione 1916 (2010) relativa alla protezione dei “whistleblower", 29 aprile 2010, http://assembly .coe.int/nw/xml/XRef/Xref-XML2HTML-en.asp?fileid=17852\&lang=en.

Assemblea parlamentare del Consiglio d'Europa (2015), Risoluzione 2060(2015) sul miglioramento della protezione dei “whistleblower", 23 giugno 2015, disponibile su http://assembly.coe.int/nw/xml/XRef/X2H-Xref-ViewPDF.asp?FileID=219 31\&lang=en.

Bang A. (2015), "Recent Developments in Whistleblower protection in Europe", European Yearbook on Human Rights 2015, NWV/Internsentia, 343-353.

Casadevall J., Myjer E., O’Boyle M. ed Austin A. (a cura di) (2012), Freedom of Expression: Essays in Honour of Nicolas Bratza, Consiglio d'Europa/Wolf Legal Publishers, Oisterwijk.

Coliver S. (2015), "The Right to Information and the Expanding Scope of Bodies Covered by National Laws since 1989", in P. Molnàr (ed.) (2015), Free Speech and Censorship Around the Globe, Central European University Press, Budapest New York, 187-210.

Comitato dei Ministri del Consiglio d'Europa (1981), Raccomandazione n. R (81) 19 relativa all'accesso ad informazioni detenute da autorità pubbliche, 25 novembre 1981. Disponibile su: https:/wcd.coe.int/com.instranet.InstraServlet?co mmand $=$ com.instranet. CmdBlobGet $\&$ InstranetImage $=600652 \&$ SecMode $=1 \& D$ ocId $=673752 \&$ Usage $=2$.

Comitato dei Ministri del Consiglio d'Europa (2002), Raccomandazione $\operatorname{Rec}(2002) 2$ relativa all'accesso all'informazione, 21 febbraio 2002. Disponibile su: https://wcd.coe.int/ ViewDoc.jsp?id=262135\&Site=CM\&BackColorInternet= C3C3C3\&BackColorIntranet=EDB021\&BackColorLogged=F5D383.

Comitato dei Ministri del Consiglio d'Europa (2011), Dichiarazione del Comitato dei Ministri relativa alla protezione della libertà di espressione e alla protezione della libertà di riunione e di associazione per le piattaforme internet gestite da operatori privati e fornitori di servizi online, 7 dicembre 2011. Disponibile su: https://wcd.coe.int/ViewDoc.jsp?id=1883671\&Site=CM\&BackColorInternet=C3 C3C3\&BackColor-Intranet=EDB021\&BackColorLogged=F5D383.

Comitato dei Ministri del Consiglio d'Europa (2014), Raccomandazione CM/ $\operatorname{Rec}(2014) 7$ relativa alla protezione dei whistleblower, 30 April 2014. Disponibile su: https://wcd.coe.int/ ViewDoc.jsp?id=2188855\&Site=CM.

Comitato per i diritti umani delle Nazioni Unite (2011), Commento generale n. 34 $\mathrm{CCPR} / \mathrm{C} / \mathrm{GC} / 34$, relativo alla libertà di opinione e di espressione (Article 19 ICCPR), 12 settembre 2011, n. 18.

Commissario per i diritti umani (2011), Human Rights in a Changing Media Landscape, Council of Europe Publishing, Strasburgo. 
Darbishire H. (2015), “A Right Emerges: The History of the Right of Access to Information and Its Link with Freedom of Expression”, in P. Molnàr (a cura di) (2015), Free Speech and Censorship Around the Globe, Central European University Press, Budapest - New York, 167-185.

Harris D. J., O’Boyle M., Bates E. P. e Buckley C. M. (2009), Law of the European Convention on Human Rights, Oxford University Press, Oxford.

Hins W. e Voorhoof D. (2007), "Access to State-Held Information as a Fundamental Right under the European Convention on Human Rights," European Constitutional Law Review 3 (2007): 114-126.

Junod V. (2009), "La liberté d'expression du whistleblower", Revue trimestrielle de droits de l'homme, 227-260.

Stevenson P. and Levi M. (2012), "The protection of whistleblowers, A study on the feasibility of a legal instrument for the protection of whistleblowers", study for the European Committee on Legal Co-operation (CDCJ), CDCJ(2012)9FIN. Disponibile su: http://www.coe.int/t/dghl/standardsetting/cdcj/Whistleblowers/ CDCJ\%282012\%299E_Final.pdf.

Tiilikka P. (2013), "Access to Information as a Human Right in the Case Law of the European Court of Human Rights", Journal of Media Law 5 (2013): 79-103.

Voorhoof D. (2014a), The right to freedom of expression and information under theEuropean Human Rights system: towards a more transparent democratic society, EUI Working paper, EUI RSCAS 2014/12 - Centro per il pluralism e la libertà dei media, Istituto Universitario Europeo, Firenze.

Voorhoof D. (2014b), "Finnish journalist's arrest, detention, prosecution and conviction for disobeying a police order during a demonstration does not violate Article 10", Strasbourg Observers Blog, 24 marzo 2014. Disponibile su: http://str asbourgobservers.com/2014/03/24/finnish-journalists-arrest-detention-prosecutio $\mathrm{n}$-and-conviction-for-disobeying-apolice-order-during-a-demonstration-does-notviolate-article-10/.

Voorhoof D. (2014c), "Whistleblower protection for journalist who alarmed public opinion about censorship on TV", Strasbourg Observers Blog, 25 novembre 2014. Disponibile su: http://strasbourgobservers.com/2014/11/25/whistleblower-protec tion-for-journalistwho-alarmedpublic-opinion-about-censorship-on-tv/\#more-26 98.

Voorhoof D. (2015a), "Freedom of Expression, Media and Journalism under the European Human Rights System: Characteristics, Developments, and Challenges”, in Molnàr P. (a cura di), Free Speech and Censorship Around the Globe, Central European University Press, Budapest - New York, 59-104.

Voorhoof D. (2015b), "Freedom of Expression and Information and the Case Law of the European Court of Human Rights and the Court of Justice of the EU. Overview and highlights 2014". Conference Paper, Justice for Free Expression in 2014, A review of global freedom of expression jurisprudence in 2014, Columbia University, New York. Available at: http://globalfreedomofexpression .columbia.edu/publications/freedom-of-gexpression-and-information-and-the-cas e-law-of-the-european-courtof-human-rights-and-the-court-of-justice-of-the-eu/. 
Voorhoof D. (2015c), "Delfi AS v. Estonia: Grand Chamber confirms liability of online news portal for offensive comments posted by its readers", Strasbourg Observers Blog, 18 giugno 2015. Disponibile su: http://strasbourgobservers.com/2015/0 6/18/delfi-as-v-estonia-grand-chamber-confirms-liability-of-online-news-portal-for -offensive-comments-posted-by-its-readers/\#more-2891.

Voorhoof D. e Gombeer T. (2008), "Klokkenluiden bij politie en justitie is uitoefening van expressievrijheid", Vigiles, Tijdschrift voor politierecht/Revue du droit depolice, 245-259.

Voorhoof D. e Humblet P. (2013), "The Right to Freedom of Expression in the Workplace under Article 10 ECHR", in Dorssemont F., Lörcher K. and Schömann I. (a cura di) (2013), The European Convention on Human Rights and the Employment Relation, Hart Publishing, Oxford, pp. 237-286.

\section{Giurisprudenza della Corte europea dei diritti dell'uomo}

Abmet Yuldırm c. Turchia, Ricorso n. 3111/10, 25 giugno 2013

Alithia Publishing Company Ltd. e Constantinides c. Cipro, Ricorso n. 17550/03, 22 maggio 2008

Appleby et al. c. Regno Unito, Ricorso n. 44306/98, 6 maggio 2003

Armellini et al. c. Austria, Ricorso n. 14134/07, 15 aprile 2015

Backes c. Lussemburgo, Ricorso n. 24261/05, 8 luglio 2008

Bladet Tromso e Stensaas c. Norvegia [GC], Ricorso n. 21980/93, 20 maggio 1999

Brosa c. Germania, Ricorso n. 5709/09, 17 aprile 2014

Brunet-Lecomte et al. c. Francia, Ricorso n. 42117/04, 5 febbraio 2009

Bucur e Toma c. Romania, Ricorso n. 40238/02, 8 gennaio 2013

Centro Europa 7 S.r.l. e Di Stefano c. Italia [GC], Ricorso n. 38433/09, 7 giugno 2012

Cetin e Şakar c. Turchia, Ricorso n. 57103/00, 20 settembre 2007

Chauvy c. Francia, Ricorso n. 64915/01, 29 giugno 2004

Cihan Ozturk c. Turchia, Ricorso n. 17095/03, 9 giugno 2009

Ciuvică c. Romania, Ricorso n. 29672/05, 15 gennaio 2013

Colombani et al. c. Francia, Ricorso n. 51279/99, 25 giugno 2002

Constantinescu c. Romania, Ricorso n. 28871/95, 27 giugno 2000

Corneliu Vadim Tudor c. Romania, Ricorso n. 6928/04 e 6929/04, 15 giugno 2006 (decisione)

Cuc Pascu c. Romania, Ricorso n. 36157/02, 6 settembre 2008

Cumpănă e Mazăre c. Romania [GC], Ricorso n. 33348/96, 17 dicembre 2004

Dalban c. Romania, Ricorso n. 28114/95, 28 settembre 1999

Dammann c. Switzerle, Ricorso n. 77551/01, 25 aprile 2006

De Diego Nafria c. Spagna, Ricorso n. 46833/99, 14 marzo 2002

De Haes e Gijsels c. Belgio, Ricorso n. 19983/92, 24 febbraio 1997 
Delfi AS c. Estonia [GC], Ricorso n. 64569/09, 10 ottobre 2013

Diche et al. c. Austria, Ricorso n. 29271/95, 26 febbraio 2002

Dink c. Turchia, nn. 2668/07, 6102/08, 30079/08, 7072/09 e 7124/09, 14 settembre 2010

Du Roy e Malaurie c. Francia, Ricorso n. 34000/96, 3 ottobre 2000

Dunca e SC Nord Vest Press SRL c. Romania, Ricorso n. 9283/05, 20 novembre 2012 (decisione)

Dupuis et al. c. Francia, Ricorso n. 1914/02, 7 giugno 2007

Dyundin c. Russia, Ricorso n. 37406/03, 14 ottobre 2008

Erla Hlynsdottir c. Icele (n. 3), Ricorso n. 54145/10, 2 giugno 2015

Ernst et al. c. Belgio, Ricorso n. 33400/96, 15 luglio 2003

Falter Zeitschriften GmbH c. Austria, Ricorso n. 3540/04, 8 febbraio 2007 (decisione)

Fatullayev c. Azerbaigian, Ricorso n. 40984/07, 22 aprile 2000

Financial Times Ltd. et al. c. Regno Unito, Ricorso n. 821/03, 15 dicembre 2009

Flux e Samson c. Moldavia, Ricorso n. 28700/03, 23 ottobre 2007

Flux c. Moldavia (n. 6), Ricorso n. 22824/04, 29 luglio 2008

Folea c. Romania, Ricorso n. 34434/02, 14 ottobre 2008

Frankovicz c. Pole, Ricorso n. 53025/99, 16 dicembre 2008

Fressoz e Roire c. Francia [GC], Ricorso n. 29183/95, 21 gennaio 1999

Friedrich Weber c. Germania, Ricorso n. 70287/11, 6 gennaio 2015 (decisione)

Fuentes Bobo c. Spagna, Ricorso n. 39293/98, 29 febbraio 2000

Gaskin c. Regno Unito, Ricorso n. 10454/93, 7 luglio 1989

Gaudio c. Italia, Ricorso n. 43525/98, 21 febbraio 2002 (decisione)

Gillberg c. Svezia [GC], Ricorso n. 41723/06, 3 aprile 2012

Godlevskiy c. Russia, Ricorso n. 14888/03, 23 ottobre 2008

Gongadze c. Ucraina, Ricorso n. 34056/02, 8 novembre 2005

Goodwin c. Regno Unito [GC], Ricorso n. 17488/90, 27 marzo 1996

Guerra et al. c. Italia, Ricorso n. 14967/89, 9 febbraio 1998

Guja c. Moldavia [GC], Ricorso n. 14277/04, 12 febbraio 2008

Guseva c. Bulgaria, Ricorso n. 6987/07, 28 novembre 2013

Haldimann et al. c. Switzerle, Ricorso n. 21830/09, 24 febbraio 2015

Heyside c. Regno Unito, Ricorso n. 5493/72, 7 dicembre 1976

Hashman e Harrup c. Regno Unito [GC], Ricorso n. 25594/94, 25 novembre 1999

Heinisch c. Germania, Ricorso n. 28274/08, 21 luglio 2011

Hertel c. Switzerle, Ricorso n. 25181/94, 25 agosto 1998

Hyde Park et al. c. Moldavia (nn. 5-6), Ricorso nn. 6991/08 e 15084/08, 14 settembre 2010

Jonina Benediktsdottir c. Islanda, Ricorso n. 38079/06, 16 giugno 2009 (decisione)

Juppala c. Finlanda, Ricorso n. 18620/03, 2 dicembre 2008 
Kania e Kittel c. Polonia, Ricorso n. 35105/04, 21 giugno 2011

Karhuvaara e Iltalebti c. Finlandia, Ricorso n. 53678/00, 16 novembre 2004

Kasabova c. Bulgaria, Ricorso n. 22385/03, 19 aprile 2011

Kayasu c. Turchia, Ricorso nn. 64119/00 e 76292/01, 13 novembre 2008

Kenedi c. Ungheria, Ricorso n. 31475/05, 26 maggio 2009

Khurshid Mustafa e Tarzibachi c. Svezia, Ricorso n. 23883/06, 16 dicembre 2008

Krone Verlag GmbH \& Co c. Austria (n. 5), Ricorso n. 9605/03, 14 novembre 2008

Kudeshkina c. Russia, Ricorso n. 29492/05, 26 febbraio 2009

Kudrevičius et al. c. Lituania, Ricorso n. 3753/05, 26 novembre 2013 (relativa alla Grande Camera))

Kydonis c. Grecia, Ricorso n. 24444/07, 2 aprile 2009

Laszlo Keller c. Ungheria, Ricorso n. 33352/02, 4 aprile 2006 (decisione)

Lavric c. Romania, Ricorso n. 22231/05, 14 gennaio 2014

Leeer c. Svezia, Ricorso n. 9248/81, 26 marzo 1987

Leempoel e S.A. Cine Revue c. Belgio, Ricorso n. 64772/01, 9 novembre 2006

Mamere c. Francia, Ricorso n. 12697/03, 7 novembre 2006

marzoenko c. Ucraina, Ricorso n. 4063/04, 19 febbraio 2009

Marin c. Romania, Ricorso n. 30699/02, 3 febbraio 2009

Martin et al. c. Francia, Ricorso n. 30002/08, 12 aprile 2012

Matuz c. Ungheria, Ricorso n. 73571/10, 21 ottobre 2014

McVicar c. Regno Unito, Ricorso n. 46311/99, 7 maggio 2002

Mihaiu c. Romania, Ricorso n. 42512/02, 4 novembre 2008

Morice c. Francia [GC], Ricorso n. 29369/10, 23 aprile 2015

Mosley c. Regno Unito, Ricorso n. 48009/08, 10 maggio 2011

Nagla c. Lettonia, Ricorso n. 73469/10, 16 luglio 2013

Nilsen e Johnsen c. Norvegia, Ricorso n. 23118/93, 25 novembre 1999

Nordisk Film \& TV A/S c. Danimarca, Ricorso n. 40485/02, 8 dicembre 2005 (decisione)

Novaya Gazeta e Borodyanskiy c. Russia, Ricorso n. 14087/08, 28 marzo 2013

Ojala e Etukeno Oy c. Finlandia, Ricorso n. 69939/10, 14 gennaio 2014

Olga Borisovna Kudeshkina c. Russia (n. 2), Ricorso n. 2827/11, 12 marzo 2015 (decisione)

Open Door e Dublin Well Women c. Irlanda, Ricorso nn. 14234/88 e 14235/88, 29 ottobre 1992

Osterreichische Vereinigung zur Erhaltung, Starkung und Schaffung eines wirtschaftlich gesunden le- und forstwirtschaftlichen Grundbesitzes c. Austria, Ricorso n. 39534/07, 17 febbraio 2015

Ozgur Gundem c. Turchia, Ricorso n. 23144/93, 16 marzo 2000

Pasko c. Russia, Ricorso n. 69519/01, 22 ottobre 2009

Pedersen e Baadsgaard c. Danimarca, Ricorso n. 49017/99, 17 dicembre 2004 
Peev c. Bulgaria, Ricorso n. 64209/01, 26 luglio 2007

Pentikainen c. Finlandia, Ricorso n. 11882/10, 4 febbraio 2014

Perna c. Italia [GC], Ricorso n. 48898/99, 6 maggio 2003

Petrina c. Romania, Ricorso n. 78060/01, 14 ottobre 2008

Pinto Coelho c. Portogallo, Ricorso n. 28439/08, 28 giugno 2011

Prager e Oberschlick c. Austria, Ricorso n. 15974/90, 26 aprile 1995

Radio Francia c. Francia, Ricorso n. 53984/00, 30 marzo 2004

Radio Twist c. Slovacchia, Ricorso n. 62202/00, 19 dicembre 2006

Ressiot et al. c. Francia, Ricorso nn. 15054/07 e 15066/07, 28 giugno 2012

Roche c. Regno Unito [GC], Ricorso n. 32555/96, 19 ottobre 2005

Roemen e Schmit c. Lussemburgo, Ricorso n. 51772/99, 23 febbraio 2003

Roşiianu c. Romania, Ricorso n. 27329/06, 24 giugno 2014

Rubins c. Lettonia, Ricorso n. 79040/12, 13 gennaio 2015

Rumyana Ivanova c. Bulgaria, Ricorso n. 36207/03, 14 febbraio 2008

Ruusunen c. Finlandia, Ricorso n. 73579/10, 14 gennaio 2014

R ůžovy panter, o.s. c. Repubblica Ceca, Ricorso n. 20240/08, 2 febbraio 2012

Saint-Paul Lussemburgo S.A. c. Lussemburgo, Ricorso n. 26419/10, 18 aprile 2013

Salumaki c. Finlandia, Ricorso n. 23605/09, 29 aprile 2014

Sanoma Uitgevers BV c. Paesi Bassi [GC], Ricorso n. 38224/03, 14 settembre 2010

Schwabe e M.G. c. Germania, Ricorso nn. 8080/08 e 8577/08, 1 dicembre 2011

Sdruženi Jihočeske Matky c. Repubblica Ceca, Ricorso n. 19101/03, 10 luglio 2006 (decisione)

Šečičc. Croazia, Ricorso n. 40116/02, 31 maggio 2007

Selisto c. Finlandia, Ricorso n. 56767/00, 16 novembre 2004

Shapovalov c. Ucraina, Ricorso n. 45835/05, 31 luglio 2012

Sosinowska c. Polonia, Ricorso n. 10247/09, 18 ottobre 2011

Steard Verlags GmbH c. Austria (n. 3), Ricorso n. 34702/07, 10 gennaio 2012

Steel et al. c. Regno Unito, Ricorso n. 24838/94, 23 settembre 1998

Stichting Ostade Blade c. Paesi Bassi, Ricorso n. 8406/06, 27 maggio 2014 (decisione)

Stoll c. Svizzera [GC], Ricorso n. 69698/01, 10 dicembre 2007

Sunday Times c. Regno Unito (n. 1), Ricorso n. 6538/74, 26 aprile 1979

Taranenko c. Russia, Ricorso n. 19554/05, 15 maggio 2014

Tarsasag a Szabadsagjogokert c. Ungheria, Ricorso n. 37374/05, 14 aprile 2009

Tatar e Faber c. Ungheria, Ricorso nn. 26005/08 e 26160/08, 12 giugno 2012

Telegraaf Media Nederle Leelijke Media N.V. et al. c. Paesi Bassi, Ricorso n. 39315/06, 22 novembre 2012

Thoma c. Lussemburgo, Ricorso n. 38432/97, 29 marzo 2001

Thorgeir Thorgeirson c. Islanda, Ricorso n. 13778/88, 25 giugno 1992

Tierbefreier E.V. c. Germania, Ricorso n. 45192/09, 16 gennaio 2014 
Tillack c. Belgio, Ricorso n. 20477/05, 27 novembre 2007

Timpul Info-Magazin e Anghel c. Moldavia, Ricorso n. 42864/05, 27 novembre 2007

Tomasz Wolek, Rafal Kasprow e Jacek Leski c. Polonia, Ricorso n. 20953/06, 21 ottobre 2008 (decisione)

Ukrainian Media Group c. Ucraina, Ricorso n. 72713/01, 29 marzo 2005

Uzeyir Jafarov c. Azerbaigian, Ricorso n. 542014/08, 29 gennaio 2015

Verdens Gang e Kari Aarsted Aase c. Norvegia, Ricorso n. 45710/99, 16 ottobre 2001 (decisione)

VgT Verein gegen Tierfabriken c. Svizzera (n. 1), Ricorso n. 24699/94, 28 giugno 2001 VgT Verein gegen Tierfabriken c. Svizzera (n. 2) [GC], Ricorso n. 32772/02, 30 giugno 2009

Vides Aizsardzības Klubs c. Lettonia, Ricorso n. 57829/00, 27 maggio 2004

Vittorio Sgarbi c. Italia, Ricorso n. 37115/06, 21 ottobre 2008 (decisione)

Voskuil c. Paesi Bassi, Ricorso n. 64752/01, 22 novembre 2007

Wegrzynowski e Smolczewski c. Polonia, Ricorso n. 33846/07, 16 luglio 2013

Wojtas-Kaleta c. Polonia, Ricorso n. 20436/02, 16 luglio 2009

Women on Waves c. Portogallo, Ricorso n. 31276/05, 3 febbraio 2009

Youth Initiative for Human Rights c. Serbia, Ricorso n. 48135/06, 25 giugno 2013

Ziembiński c. Polonia, Ricorso n. 46712/06, 24 luglio 2012 